Check for updates

Cite this: RSC Adv., 2020, 10, 15406

Received 26th January 2020

Accepted 3rd April 2020

DOI: $10.1039 / d 0 r a 00799 d$

rsc.li/rsc-advances

\section{Carbon and graphene quantum dots: a review on syntheses, characterization, biological and sensing applications for neurotransmitter determination}

\author{
Somayeh Tajik, ${ }^{a}$ Zahra Dourandish, ${ }^{\mathrm{b}}$ Kaiqiang Zhang, ${ }^{\text {cd }}$ Hadi Beitollahi, (D) *b \\ Quyet Van Le, (D) *e Ho Won Jang (iD *c and Mohammadreza Shokouhimehr (DD *c
}

Neuro-transmitters have been considered to be essential biochemical molecules, which monitor physiological and behavioral function in the peripheral and central nervous systems. Thus, it is of high pharmaceutical and biological significance to analyze neuro-transmitters in the biological samples. So far, researchers have devised a lot of techniques for assaying these samples. It has been found that electro-chemical sensors possess features of robustness, selectivity, and sensitivity as well as real-time measurement. Graphene quantum dots (GQDs) and carbon QDs (CQDs) are considered some of the most promising carbon-based nanomaterials at the forefront of this research area. This is due to their characteristics including lower toxicity, higher solubility in various solvents, great electronic features, strong chemical inertness, high specific surface areas, plenty of edge sites for functionalization, and versatility, in addition to their ability to be modified via absorbent surface chemicals and the addition of modifiers or nano-materials. Hence in the present review, the synthesis methods of GQDs and CQDs has been summarized and their characterization methods also been analyzed. The applications of carbon-based QDs (GQDs and CQDs) in biological and sensing areas, such as biological imaging, drug/ gene delivery, antibacterial and antioxidant activity, photoluminescence sensors, electrochemiluminescence sensors and electrochemical sensors, have also been discussed. This study then covers sensing features of key neurotransmitters, including dopamine, tyrosine, epinephrine, norepinephrine, serotonin and acetylcholine. Hence, issues and challenges of the GQDs and CQDs were analyzed for their further development.

\section{Introduction}

Neurotransmitters (NTs) are endogenous chemical products which play important roles as signal transducers between neurons or neurons and non-neuronal somatic cells via chemical synapses which lead to information exchange throughout the brain and body. These key chemicals are produced in different glands such as the pituitary, pineal and adrenal glands. NTs are naturally stored in vesicles and finally clustered

${ }^{a}$ Research Center for Tropical and Infectious Diseases, Kerman University of Medical Sciences, Kerman, Iran

${ }^{b}$ Environment Department, Institute of Science and High Technology and Environmental Sciences, Graduate University of Advanced Technology, Kerman, Iran.E-mail: h.beitollahi@yahoo.com

${ }^{\circ}$ Department of Materials Science and Engineering, Research Institute of Advanced Materials, Seoul National University, Seoul 08826, Republic of Korea. E-mail: hwjang@snu.ac.kr; mrsh2@snu.ac.kr

dJiangsu Key Laboratory of Advanced Organic Materials, Key Laboratory of Mesoscopic Chemistry of MOE, School of Chemistry and Chemical Engineering, Nanjing University, Nanjing, Jiangsu 210023, China

${ }^{e}$ Institute of Research and Development, Duy Tan University, Da Nang 550000, Vietnam.E-mail: Levanquyet@dtu.edu.vn at neuronal terminals. ${ }^{1}$ NTs are released from synapses followed by an action potential and pass through the synaptic gap and bind to receptors at the surface of other neurons or cells and stimulate them. Accordingly, the axon terminal of the other neuron is also activated and releases NTs similarly in order to develop communication with the adjacent neuron. Therefore, neurons show biological response leading to a complex cascade. The first neurotransmitter was discovered in 1921 and it was followed by identification of hundreds of chemical messengers in synaptic transmission. ${ }^{2}$

Among the various NTs, the biogenic amines including dopamine, epinephrine, norepinephrine, serotonin and amino acids primarily such as tyrosine, and acetylcholine category are of potential importance.,4 NTs mediated key functions in nervous system like behavior and cognition functions. They influence and regulate the muscle tone, rate of the heart, sleeping, learning, consciousness, memory, appetite, as well as mood. ${ }^{5}$ Alterations in the NTs concentrations in central nervous system (CNS) is related to various physical and mental dysfunctions like Alzheimer's, Huntington's, and Parkinson's diseases, schizophrenia, epilepsy, arrhythmias, congestive 
heart failure, glaucoma, thyroid hormone deficiency, sudden infant death syndrome (SIDS), anxiety, and depression. ${ }^{6}$

Accordingly, quantitative assessment of NTs concentrations in human fluids is highly important in terms of diagnosis, monitoring disease, and therapeutic interventions. Most common techniques for detection of concentration of NTs include fluorimetry, chemiluminescence, chromatography, mass spectrometry (MS), and capillary electrophoresis (CE). Almost all of the mentioned methods have been reported to be laborious and expensive, requiring complicated pretreatment phases, though; they have high sensitivity and selectivity with low limits of detection (LOD). In addition, these methods aren't appropriate for on-site monitoring experiments. ${ }^{7-10}$

Application of electrochemical sensors for detection of NTs suggests a valuable analysis method which is cheap and represents results in a short term, and also makes possible detection of two or more substances at the same time. High accuracy and repeatability in addition to wide range of linear response, low detection limits, and real time measurements are main advantages of electrochemical sensors. Moreover, this type of sensors is applicable for on-site detection, and is possible to be combined with strong, portable, or miniaturized devices in targeted experiments in clinical and diagnostic areas. ${ }^{\mathbf{1 1 , 1 2}}$

Since appearance of chemically modified electrodes, significant improvements have been developed in electroanalysis field because of the better sensitivity and selectivity than other electrodes. Chemically modification of electrodes via immobilized material on its surface develops its unique chemical, electrochemical, electrical, and other desirable features. Notably, the metal nanoparticles (NPs) and metal oxide NPs, carbon nanotubes, biomolecules, as well as QDs are usually used for electrode modification among which, researchers mainly investigated the QDs and composites in recent years. ${ }^{\mathbf{1 3 , 1 4}}$

QDs have been considered to be a group of semi-conductor NPs which have 1 to $10 \mathrm{~nm}$ diameter and are consisted of elements from the II-VI or III-V periodic groups. In fact, the QDs have been considered as quasizero dimensional nanomaterials, while 3 dimensions have the size $\leq 10 \mathrm{~nm}$, so that the internal electrons mobility would be limited into nano-scale dimensions in each direction. There is significant interest in usage of semiconductor QDs (SQDs) and small organic fluorescent dyes (OFDs) in biomedicine and bio imaging based on the respective increased quantum yield as well as good optical features. Nonetheless, they also have some disadvantages that have limited their applicability among which higher toxicity, lower biocompatibility, expensiveness as well as lower chemical inertness can be mentioned. These materials reveal low solubility/bio-availability in water-based solution. Different methods have been suggested in order to improve solubility of QDs including locating them into a shell like silica, usage of the stable surfactant layer, substitution of the polar groups on the OFDs surface, but modification mostly made in the organic solvents. Actually, the carbon based QDs including GQDs and CQDs are usually classified as the zerodimensional substances which have fluorescence property. However, from the time of the first identification of CQDs by Sun et al., as well as the GQDs by Pan et al., ${ }^{18}$ it has been found that they are considerably more efficient than semiconductors QDs because of lower toxicity, higher biocompatibility, acceptable chemical inertness, and solubility. ${ }^{15-17}$

According to the research, graphene QDs have been proved to be derivations of the graphene/graphite and other graphitic three-dimensional materials through top down synthetic approaches. Normally G-QDs have layered structures and lateral size up to $100 \mathrm{~nm} .{ }^{18}$ In contrast, carbon dots are produced in bottom-up synthetic approaches and possess spherical shape with a diameter up to $10 \mathrm{~nm}$. Moreover, such particles have been called carbogenic dots or carbon nano-dots. In addition, the GQDs display physico-chemical features as the same as the features of the graphene. In terms of dimension, the GQDs have been considered to be little graphene sheets with lateral sizes less than $10 \mathrm{~nm}$ graphene layers, which formed the final particle. However, a series of the CQDs features diverged from graphene features as a result of predominance of the edge impacts with CQDs, quantum confinement, as well as the little sizes and biocompatibility, which cause them to be known as the efficient carriers for drug delivery, making possible concurrent visualization of the releasing kinetics. Consequently, their certain physicochemical and catalytic features provide the possibility for various biomedical applications. ${ }^{19-21}$

We first briefly discuss synthesis procedure of GQDs and CQDs. Then, their characterization methods will be described. Then, we concentrate on varieties biological and sensing applications. Furthermore, we highlight application of GQDs and CQDs based electrochemical sensors in order to determine some of important neurotransmitters.

\section{Synthetic methods}

Synthesis of CQD and GQDs can be generally classified into "top-down" and "bottom-up" methods. The former involves cleaving or breaking down of carbonaceous materials via chemical, electrochemical, or physical approaches. The latter is realized by pyrolysis or carbonization of small organic molecules or by step-wise chemical fusion of small aromatic molecules. $^{22}$ Recently, there has been much interest in the development of bottom-up approaches for the preparation of CQDs and GQDs due to the precise control of precursor molecules, ease of techniques, low cost, and practicality and convenience of the procedure with generally nontoxic precursors. ${ }^{23}$

\subsection{Synthesis of CQDs}

Authors devised a lot of techniques to procure the CQDs since introduction of CQDs. Laser ablation, arc discharge, electrochemical oxidation, ultrasonic treatment and chemical oxidation can be mentioned among top-bottom methods. However, bottom-up methods consist of pyrolytic process, template method, hydrothermal/solvothermal methods, and microwave assisted method.

Efficiency of CQDs is possible to be increased in arrangement or post-treatment steps. Modification of CQDs is also important in order for obtaining desirable surface features as main feature for utilizations and solvency. 
In addition, carbon precursors are also used to produce carbon dots, such as the ground coffee, the applied tea, grass, as well as the light sediment. Production of carbon dots using these precursors is high efficient and economic. ${ }^{24}$

\subsubsection{Top-down methods}

2.1.1.1. Laser ablation. The laser ablation method uses a high-energy laser pulse to irradiate surface of the target to a thermo-dynamic mood where creates higher temperatures and pressures, while heat increased and evaporated into a plasma mood. Finally, the vapor is crystallized into NPs. ${ }^{25,26}$

In their study, Sun et al. constructed the CQDs through the laser ablation into a carbon target in the presence of water vapour and argon as the carrier gas at $75 \mathrm{kPa}$ and $900{ }^{\circ} \mathrm{C} . \mathrm{HNO}_{3}$ reflux was applied for $12 \mathrm{~h}$ and then surface was immobilized via attachment of the simplified organic samples like $\mathrm{PEG}_{1500 \mathrm{~N}}$ (amine-terminated poly-ethylene glycol) as well as poly(propionyl ethylene eimine-coethyleneimine) (PPEI-EI) leading to the production of acid-treated CQDs with luminescence emission. $^{17}$

Doñate-Buendia synthesized fluorescent CQDs using the laser irradiation into the carbon glassy particles that have been suspended in polyethylene glycol 200 via 2 procedures, including a flow jet configuration and a batch. Therefore, CQDs synthesized via the flow jet method have a mean size of $3 \mathrm{~nm} .{ }^{27}$

$\mathrm{Hu}$ et al. revealed that surface of CQDs is possible to modify via selection of adequate organic solvents in the course of the laser irradiation which leads to adjust PL features of the synthesized CQDs. ${ }^{28}$

Simplicity has been considered as one of the advantages of laser ablation while it can lead to produce different types of nanostructures. High amount of carbon material is needed for procuring the carbon targets. Moreover, the synthesized carbon NPs dimensions using the laser irradiation completely differ because large-sized NPs are easily distributed during centrifugation, therefore efficiency of synthesis of the carbon NPs and the carbon material has been utilized in small quantity. ${ }^{29}$

2.1.1.2. Electrochemical oxidation. The electrochemical oxidation is one of the simplified methods to procure the NPs, ${ }^{30}$ but this can be conducted under normal temperature and pressure. Experts in the field reported electrochemistry technique as one of the adequate methods for synthesizing the CQDs, since it can be used to regulate particle size, higher purity, affordable, higher yield, and PL performance of synthesized CQDs.

In another study, Liu et al. dealt with synthesizing the CQDs with the average diameter of $(4.0 \pm 0.2) \mathrm{nm}$ and higher crystalinity through electrochemical oxidation of a graphite electrode in the alkaline alcohols. Interestingly, the as-established CQDs dispersion had no color and slowly its color shifted to the bright yellow after storage in room temperature that is suggested to be due to oxygenation of the surface samples. Finally, researchers demonstrated that the respective potentials as well as $\mathrm{pH}$ are key aspects in generation of CQDs. ${ }^{31}$

Deng et al. showed that alcohols with low-molecular-weight can be used as single carbon source in order to achieve controllable synthesis condition and produce fluorescent $\mathrm{C}$ dots via one-pot electrochemical method. Synthesized C-dots show high FL quantum efficiency, while various size and maximum emission wavelengths can be obtained through applying varied potential. ${ }^{32}$

2.1.1.3. Arc discharge. First fluorescent CQD was detected while $\mathrm{Xu}$ et al. purified the single-walled carbon nanotubes (SWCNTs) through an oxidation procedure between nitric acid and the arc-discharged soot. ${ }^{33}$ Additionally, Arora and Sharma stated arc discharge can be used to reorganize carbon atoms which are obtained via decomposition of bulk carbon precursors in the anodic electrode as derivation of gas plasma obtained in a sealed reactor. Therefore, temperature in the reactor could approximate $4000 \mathrm{~K}$ using electric current with the aim of production of high energy plasma. Finally, the CQDs were produced using the arc discharge procedure. ${ }^{34}$

2.1.1.4. Ultrasonic treatment. It has been found that ultrasound can be used to develop high and low pressure wave in the liquid environment, causing the generation and distribution of little vacuum bubbles. The act of cavitation leads to high speed impinging of the liquid jets, de-agglomeration and powerful hydro-dynamic shear force. Therefore, the macroscopic carbon materials can be cut into nanoscale CQDs using the energy of ultrasonic waves. ${ }^{35}$

Zhang et al. addressed synthesizing the new BiOBr/N-CD nanocomposites using straightforward ultrasonic based method in which the connection between BiOBr and nitrogencarbon dots (N-CDs) is the key element. ${ }^{36}$ Moreover, Ma et al. have introduced a simple ultrasonic based approach for synthesizing the NCDs with the use of the aqua ammonia and glucose as the precursor. NCDs shows high aqueous dispersibility with no surface modifications, that also revelas stable and robust visible emissions as well as the high upconversion PL, at the same time. ${ }^{37}$

Dang et al.'s study was the first that introduced an easy and cheap method for large scale fabrication of white fluorescent carbon dots (WCDs) through a one-phase ultrasonic procedure using polyamide resin. Finally, the CDs represented white fluorescence with quantum yield (QY) as much as $\sim 28.3 \%$ following the treatment with further ultrasonic and KH570 as the co-passivating factor. ${ }^{38}$

2.1.1.5. Chemical oxidation. Acids with strong oxidization capability adds carbon atoms into small organic molecules and converts them into carbonaceous materials which is possible to be incised into little sheets via the controlled oxidation. ${ }^{39,40}$ Although, the mentioned procedure is sensitive to severe conditions and drastic processes; however, it can improve the water solubility and fluorescence features significantly. Additionally, it makes large scale generation of the CQDs possible and utilizes the inexpensive and plentifully accessible starting substances. Peng and Travas-Sejdic introduced a novel method for synthesis of the luminescent CQDs in the aqueous solutions via de-hydrating carbohydrates using the concentrated sulfuric acid which would be accompanied by decomposing the carbonaceous substances into the single CQDs with the usage of nitric acid, then ultimately immobilization with the amineterminated compositions (4,7,10-trioxa-1,13-tridecanediamine). The surface immobilization is a significant step for the CQDs. Consequently, the emission of CQDs wave-length 
could be regulated by changing the time scale of nitric acid treatment as well as the raw substances. As a result, the multi color emission and non-toxic nature enabled these compounds for utilizing in the bioscience studies. ${ }^{41}$

The fullerene carbon soot (FCS) can converted into CQDs with high production efficiency under mild conditions using a combination of acids such as concentrated $\mathrm{HNO}_{3}$ and $\mathrm{H}_{2} \mathrm{SO}_{4}$. Fluorescence quantum efficiency obtained from as-production process of CQDs is about $3 \%$ to $5 \%$. More importantly, CQDs obtained using the mentioned procedure showed emission in the yellow range of the visible light that has been considered as an advantage for the respective potential utilizations. Moreover, other examinations revealed that the CQDs have been strongly photostable in the broad pH-range and showed appropriate resistance in the ionic strength and lengthy UV irradiation which in turn increases their usage potential in undesirable conditions. $^{42}$

\subsubsection{Bottom-up methods}

2.1.2.1. Pyrolytic process. Thermal decomposition has been known as the preference method for production of C-dots which is conducted by pyrolysis or carbonizing the carbon precursors at the increased temperatures. Advantages of the above procedure include the simplified operations, solvent-free approaches, wider precursor tolerance, shorter reaction duration, inexpensiveness, and the scalable generation. In addition, optical features of C-dots is optimized through alterations in main factors like the reaction temperatures, reflux duration, and reaction mix $\mathrm{pH} .{ }^{43}$ For example, Wang et al. synthesized CQDs with size control approach and markedly high QY (87\%) through direct carbonization of the distributed carbonaceous micro-crystals in a mesophase process. Notably, the as-prepared CQDs sizes have been regulated by controlling over nucleation temperature to form mesophase. High QY value is obtained to as-prepared CQDs because of oxygen-free nature. ${ }^{\mathbf{4 4}}$ in their study, Ma et al. dealt with synthesizing the N-doped GQDs on the basis of the graphene-like structures using the direct carbonization of the ethylene diamine tetra-acetic acid (EDTA) in a sand bath at $260-280{ }^{\circ} \mathrm{C}$. The samples generated in the course of the EDTA de-carboxylation can slowly mix with each other which results in graphite like structures at the solid-state reaction condition. Accordingly, $\mathrm{N}$-containing compositions are transformed into diverse graphitic carbon nitrides at very high pyrolysis temperature. ${ }^{45}$

2.1.2.2. Micro-wave assisted technique. According to the studies, micro-wave has been considered to be one of the electro-magnetic waves which has wave-length within the range between $1 \mathrm{~mm}$ and $1 \mathrm{~m}$, and could provide intensive energies for decomposing the chemical bonds in a substrate. Microwave technique has been regarded as a quick and affordable methods, which could effectively slow down the reaction duration and present concurrent homogeneous heating that helps produce the smooth size distribution of the QDs. ${ }^{46}$ RodríguezPadrón et al. proposed catalysis approach of a lignocellulosic raw material using microwave that results in forming little CQDs $(17.5 \mathrm{~nm})$ with certain physico-chemical features like stability, life-time fluorescence $(\tau=0.57 \mathrm{~ns}$ ) as well as the harmonic emission based on the excitation wave lengths. ${ }^{47}$
Yang et al. reported straightforward microwave based method in order to produce nitrogen doped CQDs (NCQDs) in a single phase via an ammonia solution with xylan as a precursor at $200{ }^{\circ} \mathrm{C}$ and input power of $200 \mathrm{~W}$ in 10 minutes. The N-CQDs synthesized by a green, economical and quick approach results in the increased crystal quality, desirable PL features as well as higher tolerance for the salt and metal ion. ${ }^{\mathbf{4 8}}$

Ko et al. synthesized a biocompatible silk fibroin based CQD (SF-CQD) using the microwave irradiation for the first time. In the suggested method, SF has been dissolved in the distilled water at $20 \mathrm{mg} \mathrm{mL}^{-1}$ concentration. In the following, $4 \mathrm{~mL}$ of obtained SF solution with white color has been utilized as the precursor and irradiated by microwave at $200{ }^{\circ} \mathrm{C}$ for twenty minutes. Centrifugation of the obtained yellow solution has been performed two times at $3500 \mathrm{rpm}$ for thirty minutes in order for removing the big aggregations. Then supernatant has been filtered via $0.22 \mu \mathrm{m}$ poly-ethersulfone (PES) syringe filter and finally lyophilized to achieve a yellow solid. ${ }^{\mathbf{9}}$

2.1.2.3. Template method. It should be mentioned that authors also applied the template procedure in order to synthesize the nano-sized C-dots. In fact, synthetic procedure includes 2 main phases of synthesis of the C-dots via calcining in proper mesoporous template or silicon spheres and etching for removal of the support and generation of the nano sized Cdots. Lai et al. produced carbon dots in mesoporous silica NPs which was used as a nano reactor in order to monitor the size distribution. At first place, the researchers generated mesoporous silica NPs $\left(\mathrm{mSiO}_{2}\right)$; while, the $\mathrm{mSiO}_{2}$ NPs have been mixed with the PEG- $\mathrm{NH}_{2}$ and glycerol, consequently heated at $230{ }^{\circ} \mathrm{C}$ up to thirty minutes; and ultimately the crude items have been extracted by centrifuging in order to achieve nanocomposites of carbon dots without scratching. Simultaneously, PEG was used to cap surface of $\mathrm{mSiO}_{2}$ which led to improvements in QY, biocompatibility, and colloidal stability of subsequent carbon dots. In carbon dots synthesis process, it was expected that corrosive acid or the base etch the template since forming mesoporous silica has been a bottleneck. Such an approach has been laborious and costly. Additionally, since the template pyrolysis was done only under high temperature, complete hatch off of template was hard. In addition, separation and purification steps were also difficult, and led to limitation in QY.50

Yang et al. were the first who introduced a soft-hard template procedure to producing photoluminescent CDs with elegant dimensions, composition, crystalline degree as well as the PL features. Normally, in this method, the co-polymer Pluronic P123 was used as the soft template and the ordered mesoporous silica was used as the hard template, diverse organic molecules like 1,3,5-trimethylbenzene (TMB) and diaminebenzene (DAB) were also used as the source of carbon. Upon the carbonization, the template removal and inactivation, the achieved C-dots with harmonic dimensions, compounds as well as the crystalline degrees represented higher PL throughput about 3.3 to $4.7 \%$, upconversion PL, and the extremely high stability features. In fact, the soft-hard template procedure removes aggregation issue and makes preparation of C-dots possible with narrow size distribution due to the size limits. ${ }^{51}$ 
2.1.2.4. Hydro-thermal/solvo-thermal methods. According to the studies, hydrothermal carbonization (HTC) or solvothermal carbonization has been considered an inexpensive, environmental-friendly, and non-toxic methods in order to produce the new carbon based materials from various raw substances. Normally, an organic solution is reacted as the precursor and sealed in a hydrothermal reactor via the increased temperatures. ${ }^{52}$ Therefore, the CQDs have been produced via the HTC using different raw substances like protein, glucose, citric acid, and chitosan. Shen et al. offered the CQDs synthesis via hydrothermal carbonization approach with the use of the glucose and citric acid as raw materials. Afterwards, the as-prepared CQDs have been coupled with P25 to achieve the CQDs/ $\mathrm{TiO}_{2}$ composite in hydrothermal technique at $150{ }^{\circ} \mathrm{C}$ for 6 hours. ${ }^{53}$

Zhao et al.'s study addressed the synthesis of a strongly photoluminescent N-CQDs with the QY equal to $23.1 \%$ in one phase via the hydrothermal procedure of polyacrylamide as the nitrogen and carbon origins, then they centrifuged the resultant mixture. These N-CQDs with the average diameter of $3 \mathrm{~nm}$ displayed reasonable water solubility and the fluorescence features so that they could be utilized as efficient probes for sensitively and selectively detecting dopamine (DA) ${ }^{54}$

Solvothermal carbonization and then extraction with the organic solvent has been known as one of the well-known approaches to produce the CQDs. ${ }^{55}$ In this method, carbonyielding raw materials are exposed to treat the heat in the increased boiling point organic solvents, which is accompanied by the concentration and extraction processes. Wang et al. reported a single step solvothermal method for production of highly fluorescent hollow CDs using bovine serum albumin as a reinforced carbon origin. Notably, the combination of the bovine serum albumin, ultrapure water and ethanol has been heated under the fixed temperatures $\left(180^{\circ} \mathrm{C}\right)$ for 12 hours in order to obtain a light brown solution. Centrifugation and dialysis lead to formation of HCDs with average diameter $6.8 \pm$ $1.3 \mathrm{~nm}$ and QY of 7\%. ${ }^{56}$ In their study, Bhunia et al. prepared 2 kinds of the CQDs, including the hydrophobic and hydrophilic with a diameter $<10 \mathrm{~nm}$ using the carbohydrate carbonization. In addition, the hydrophobic CQDs have been obtained via blending diverse volumes of carbohydrate with octadecene and octadecylamine prior to heating under $70-300{ }^{\circ} \mathrm{C}$ for 10 to 30 minutes. The hydrophilic CQDs were prepared via heating the aqueous solution consisting of the carbohydrate with a wider range at $\mathrm{pH}$. Therefore, hydrophilic CQDs have yellow and red emissions and could be made via blending the aqueous solution of carbohydrate with the concentrated phosphoric acid, followed by heating at $80-90{ }^{\circ} \mathrm{C}$ for sixty minutes. ${ }^{55}$

\subsection{Synthesis of GQDS}

Considering the latest construction procedures of the GQDS which have been published in the literature, methods of GQDs synthesis could be categorized into 2 main groups, including the bottom-up and top-down production methods. Therefore, these top-down procedures, which are more used in the nanoscience are applied by incising the big graphene sheet, CNTs, carbon fibers or the graphite into the little segments of graphene sheets which have been counted as the most appropriate methods for the mass generation. These approaches include electrochemical exfoliation, ultrasonication exfoliation, acidic oxidation, electron beam lithography, and chemical exfoliation. However, the bottom-up methods consist of QDs synthesis using poly-cyclic aromatic compositions or molecules with the aromatic structures like fullerenes via pyrolysis or carbonization, cage-opening of fullerene, microwave, hydrothermal, solvothermal, and template methods. ${ }^{24,57}$

\subsubsection{Top-down methods}

2.2.1.1. Electrochemical exfoliation. He et al. produced coal based GQDs using regular micro variation in size via electrochemical exfoliation method. Fig. 1 depicts the whole construction procedure of the coal based GQDs. ${ }^{58}$ The researchers used a platinum plate electrode as a counter electrode and a little cuboid coke lump was applied as a working electrode. Then, the coke has been electrolyzed in the $\mathrm{H}_{2} \mathrm{O}$ and $\mathrm{MeOH}$ mixture at distinct ratios of volume in the range between $10: 90$ and $85: 15$ with $0.1 \mathrm{M}$ of $\left(\mathrm{NH}_{4}\right)_{2} \mathrm{~S}_{2} \mathrm{O}_{8}(\mathrm{pH}$ equal to 7.0) which is considered as the electrolyte for one hour, and the current intensity in the range between 80 and $400 \mathrm{~mA} \mathrm{~cm}^{-2}$. Afterwards, samples of color GQD were collected using centrifuge and dialysis. In the following, adjusting $\mathrm{H}_{2} \mathrm{O}$ volume in the electrolyte solution and the current density was used to the coke, while the Y-, O-, and G-GQDs had the size between 3.02 and $4.61 \mathrm{~nm}$ with the increased QY of $9.24 \%, 7.90 \%$ as well as $8.47 \%$. In addition, the bright B-GQDs were obtained with the QY as much as $19.27 \%$ through reducing $\mathrm{NaBH}_{4} \cdot{ }^{58}$

Fu et al. devised an electrochemical synthesis technique for preparing the N-GQDs with average diameter of $20 \mathrm{~nm}$. These NPs can release 3 basic color fluorescence of blue, red, and green independently because of their quantum confinement and the edge impacts of the surface moods. The as-procured NGQDs have been utilized for specifically and sensitively detecting the $\mathrm{Fe}^{3+}$ ion with the broader linear range between 0 and $200 \mathrm{mM}$ and lower LOD equal to $0.87 \mathrm{mM}^{59}$

2.2.1.2. Ultrasonication exfoliation. According to the above technique, the GQDs have been procured through one-phase process with any need for the costly equipment or specific

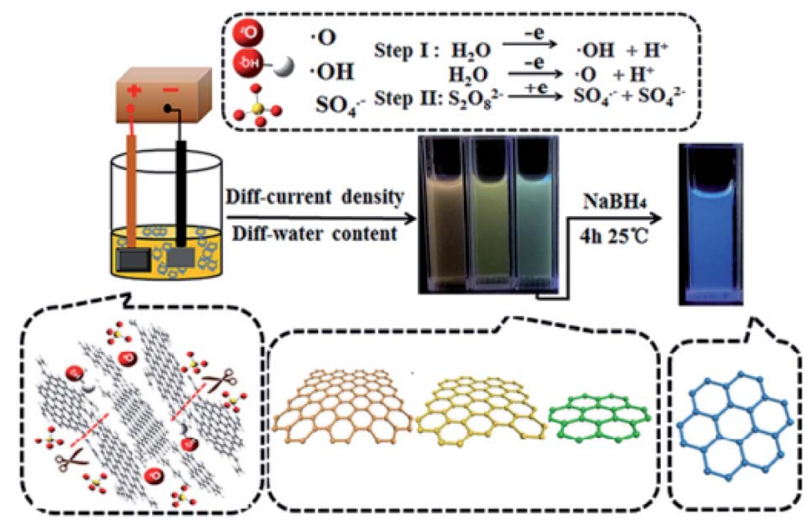

Fig. 1 A schema of the synthetic procedure of multi-color GQDs from the coke. Reprinted with permission. ${ }^{58}$ Copyright 2018, Elsevier. 
conditions. Zhuo et al. revealed GQDs procured by the ultrasonic approach. In this approach, graphene has been oxidized in the concentrated $\mathrm{HNO}_{3}$ and $\mathrm{H}_{2} \mathrm{SO}_{4}$ solutions at the room temperature for twelve hours. Afterwards, the solution mixture experienced an ultrasonic treatment for twelve hours with an ultrasonic device. Then, the obtained mixture was calcinated at $350{ }^{\circ} \mathrm{C}$ for twenty minutes in order to discard the concentrated $\mathrm{HNO}_{3}$ and $\mathrm{H}_{2} \mathrm{SO}_{4}$. It should be noted that the as-procured products have been re-dispersed in water. Next, a $0.22 \mu \mathrm{m}$ microporous membrane has been used to filter the obtained black suspension in order to obtain a brown filter solution. Finally, the solution has been additionally dialyzed to obtain GQDs. ${ }^{60}$

2.2.1.3. Acidic oxidation. A simple synthesis approach of GQDs in large scale was introduced by Peng et al. using the acidic exfoliation and etching of the pitch carbon fibers. The asprocured GQDs, had a dimension ranging between 1 and $4 \mathrm{~nm}$, and exhibited two dimensional (2D) morphology. Based on the outputs, synthesis temperature impacted the colour of the PL emission. ${ }^{61}$ Moreover, a recent study of Iannazzo revealed that Pristine MWCNT has been utilized as a precursor to procure the GQDs. In addition, the pristine MWCNT had reaction with the $\mathrm{HNO}_{3} / \mathrm{H}_{2} \mathrm{SO}_{4}$ mix (1:3 ratio). Therefore, sonication of the obtained mix has been performed in an ultrasonic water bath at $60{ }^{\circ} \mathrm{C}$ for 4 days. Next, dilution and filtration of the reaction mix has been done at vacuum condition; $\mathrm{NaOH}$ has been used to neutralize the filtrate and centrifugation has been done at $3000 \mathrm{rpm}$. Notably, the deionized water has been used to wash the final compounds many times, and the deionized water has been exploited to dilute the obtained brown powdery mixture. Then, it has been dialyzed for eight hours. The resultant product has been gathered; finally dried under vacuum at $60{ }^{\circ} \mathrm{C}$ to achieve pure GQDs. ${ }^{62}$

2.2.1.4. Electron beam lithography technique. According to the studies, the electron beam lithography procedure has been introduced for the first time to procure the GQDs. ${ }^{63}$ Nevertheless, the method has no widespread utilization as a result of the required costly instrumentation. In addition, GQDs size has been restricted by the scale of the lithography technology process and the GQDs diameter has been arbitrarily modified. However, the above technique developed one of the crucial fields in $0 D$ carbon substances that is the cause for the increased excitement into the academic field.

Lee et al. reported an approach for the size-controlled fabrication of the smooth GQDs with the use of the self assembled block copolymers (BCP) which are used as the etch mask on the graphene films increased by the chemical vapor deposition (CVD). Even though the above technique obtained lower yield, but this condition caused the highly smooth particles to probe the impacts of the functionalization and size. ${ }^{64}$

2.2.1.5. Chemical exfoliation. Sapkota and coworkers utilized a 2-phase liquid-phase chemical exfoliation procedure in order to synthesize GQDs which is a scale up method and results in big amounts of the exfoliated substance. In fact, the graphite dark-brown dispersion is achieved in Step 1 of the synthesis procedure and at Step 2 and a dialysis-based purification phase, it turns to a golden-colour. ${ }^{65}$ besides, Biswas et al. report a 1-phase, affordable, aqueous-phase, the simplified chemical exfoliation procedure to completely convert the multiwall carbon nanotubes to the water dispersible GQDs which yielded the average dimension equal to $\sim 3 \mathrm{~nm}$ with the use of the sodium bismuthate $\left(\mathrm{NaBiO}_{3}\right)$ as one of the robust oxidants. ${ }^{66}$

\subsubsection{Top-down methods}

2.2.2.1. Pyrolysis or carbonization. Hong et al. reported a novel procedure to make the monolayer GQDs with the ultrasmall lateral dimension equal to $1.3 \pm 0.5 \mathrm{~nm}$ which involved pyrolysing the trisodium citrate as well as the subsequent ultra filtration. ${ }^{67}$

In a characteristic synthesis, $0.4 \mathrm{~g}$ of the dried VCX-72 carbon black has been refluxed in $100 \mathrm{~mL}$ of $\mathrm{HNO}_{3}$ for 24 hours. In addition, an ultrasonic cell crusher has been used to treat the suspension at $950 \mathrm{~W}$ for ten minutes and then centrifuging has been done $(8000 \mathrm{rpm})$ for ten minutes which led to get the supernatant. Then, filtration of the supernatant has been done by a $0.22 \mu \mathrm{m}$ micro-membrane filter. Acid has been removed using rotary evaporation. Afterwards, the dried material has been dissolved in $10 \mathrm{~mL}$ of DI water. Next, it has been dialyzed in 3500 Da dialysis bag for one day. Consequently, the freezedrying method has been also obtained for getting the GQD powder. In pyrolysis techniques, diverse little organic molecules could be chosen as the raw substances. As a result, the mentioned procedures have been affordable, and also yield exact dimension control, allowing the natural inheritance of the heteroatoms from the precursors. ${ }^{68}$

2.2.2.2. Cage opening of fullerene. Kaciulis et al. added fullerene to the mix of the sodium nitrate, potassium permanganate, as well as concentrated sulfuric acid to fabricate GQDs as fluorescent sensor. ${ }^{69}$ For example, Lu et al. obtained highly little GQDs using ruthenium catalyzed cage opening of $\mathrm{C}_{60}$. The ruthenium surface makes strong interactions with the $\mathrm{C}_{60}$ molecules that caused the surface vacancy on the ruthenium, helping the $\mathrm{C}_{60}$ molecules for embedding into the surface. Embedded molecules are fragmented at the enhanced temperature and then carbon clusters are produced, which undergo aggregation and diffusion for forming the GQDs. Thus, the GQDs shape or form can be adjusted via optimization of the annealing temperature. ${ }^{70}$

2.2.2.3. Microwave methods. Li et al. introduced a simple, superfast, and scalable approach for production of the GQDs in three minutes under micro-wave irradiation (MA-GQDs). Therefore, MA-GQDs showed good fluorescence quantum with efficiency up to $35 \%$. In addition, the ultra bright fluorescence as well as stable MAGQDs can be used as a fluorescence probe and phosphor for developing the white light emitting diodes and the cell imaging fields. ${ }^{71}$ Also Kumawat et al. developed a simplified one-pot microwave assisted green synthesis approach for production of the bright red luminescent GQDs with the use of the ethanolic extracts of Mangifera indica (mango) leaves, and therefore reflecting them as the mGQDs. In addition, mGQDs had quantum-size in the range between 2 and $8 \mathrm{~nm}$ and demonstrated excitation in related fluorescence emissions in the near infrared area from 650 to $750 \mathrm{~nm} .^{72}$ Additionally, the synthesis of the FGQDs has been done using a microwave assisted hydrothermal procedure, and hydrofluoric acid (HF) and glucose as a precursor substance. To sum up, addition of $4 \mathrm{~mL}$ of 


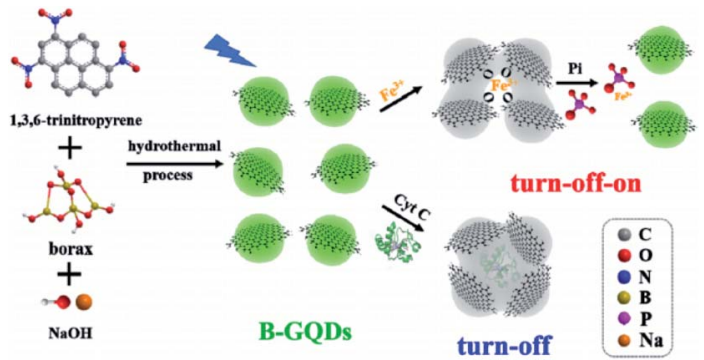

Fig. 2 A schema of the one-phase procurement of the B-GQDs to make the sensors (toward the $\mathrm{Fe}^{3+}$ ion and phosphate-Pi) as well as the bio-sensor (toward the cytochrome C-Cyt C). Reprinted with permission. ${ }^{74}$ Copyright 2019, Elsevier.

the HF acid into the glucose solution has been done. The whole reaction mixture placed in autoclaves and microwave as hydrothermal reactor at $180{ }^{\circ} \mathrm{C}$ and stored for nearly three hours. The product was filtered through $0.2 \mu \mathrm{m}$ filter and $\mathrm{pH}$ was neutralized with using $\mathrm{NaOH}$. Resulted light brown solution has been dialyzed with the help of the dialysis bag. ${ }^{73}$

2.2.2.4. Hydrothermal and solvothermal methods. A one phase bottom-up molecular fusion approach between the borax (dopant) and 1,3,6-trinitropyrene (carbon source) in the sodium hydroxide (alkaline medium) was used to produce B-GQDs under hydrothermal condition (Fig. 2). In this approach, dispersion of the borax $(0.131 \mathrm{M})$ and 1,3,6-trinitropyrene $\left(2 \mathrm{mg} \mathrm{mL}^{-1}\right)$ has been performed in the $\mathrm{NaOH}$ solution $(0.125 \mathrm{M}, 100 \mathrm{~mL})$ with an ultrasonic treatment for 0.5 hours prior to transferring it to the hydrothermal autoclave for hydrothermal reactions. Therefore, the obtained brown solution has been dialyzed with the use of the dialysis bags. Moreover, saffron yellow solution of B-GQDs was observed following the dialysis and then freeze-dried. Finally BGQDs were applied as sensitive detector of $\mathrm{Fe}^{3+}$ or $\mathrm{Fe}^{3+}$-containing protein..$^{74}$

Ko et al. used citric acid and ethylene diamine as the carbon origin in order to produce GQDs by hydrothermal method. In this method, dissolution of the citric acid and ethylene diamine has been done in $5 \mathrm{~mL}$ de-ionized water. Then, it has been shaken to obtain a transparent solution. Next the solution has been transported into a $20 \mathrm{~mL}$ Teflon lined stainless steel autoclave. Afterwards, heating the sealed autoclave has been performed to $160^{\circ} \mathrm{C}$ for $4 \mathrm{~h}$. In addition, the resultant product has been gathered after addition of ethanol in the solution and then centrifuging has been performed at $5000 \mathrm{rpm}$ for five minutes. Finally, the solution has been re-dispersed in to water. ${ }^{75}$

2.2.2.5. Template method. Li et al. procured the GQDs using a soft template approach. In fact, 1,3,5-triamino-2,4,6trinitrobenzene (TATB) that has been a planar and strongly symmetric molecule with 6 powerful intramolecular hydrogen bonds between $-\mathrm{NH}_{2}$ and $-\mathrm{NO}_{2}$ functional groups. It also has a graphitic like layered structure which has been utilized as a carbon template and precursor. At the first step, the TATB has been annealed in a thermal procedure leading to the breakdown of diverse chemical bonds, along with the expanding gases formation; for example, $\mathrm{NO}_{2}, \mathrm{NO}$, and $\mathrm{H}_{2} \mathrm{O}$. Consequently, multilayer graphitic like TATB was converted into the single layer compounds owing to the exposure to the expanding gas. At the end, the oxidative exfoliation has been applied to achieve the dispersed N-GQDs. However, a main advantage of soft template method has been the uniform size of GQDs. ${ }^{76}$

In their study, Gao et al. used little molecular carbon disulphide as the precursor to produce the GQDs doped with sulphur. Then, the mix liquid has been burnt for the formation of the carbon powder, which easy the exfoliated to GQDs in the next step. The GQDs smoothness as well as the dispersity have been described by transmission electron microscope (TEM), reflecting the average diameter equal to $2.46 \mathrm{~nm} .{ }^{77}$

\section{Characterization of $C Q D$ and GQD}

Considering the importance of collecting the data of the synthetic features of the CDs and GDs, multiple procedures can be exploited for the characterization of the CQDs, as an instance, the nuclear magnetic resonance (NMR), TEM, X-ray diffraction (XRD), fourier transform infrared spectroscopy (FTIR), photoluminescence, UV spectroscopy and Raman spectroscopy.

\subsection{Transmission electron microscope}

TEM possesses a high-resolution as much as $0.1-0.2 \mathrm{~nm}$ which could be utilized for identifying the sample ultra structure. Therefore, the procedure could be used for the NPs morphological studies, providing results for understanding their shapes, sizes, dispersion, and so forth. In fact, TEM has been widely used as one of the parts of description of the CQD and GQD. ${ }^{78-80}$ In order to

A
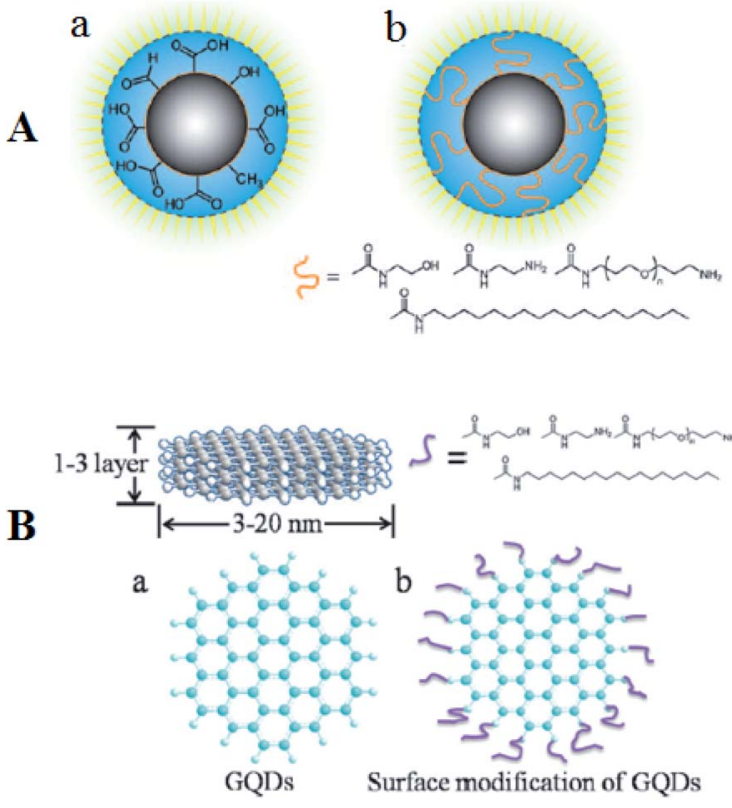

Fig. 3 (A) Depiction of C-dots (a) following the treatment of surface oxidative and (b) following functionalizing with the surface-passivation reagent. (B) (a) A schema of the GQDs and (b) structural patterns following the functionalization with the surface-passivation reagents. Reprinted with permission. ${ }^{17}$ Copyright 2006, American Chemical Society. 
a

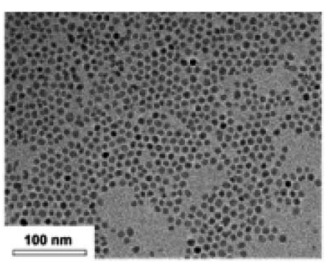

b

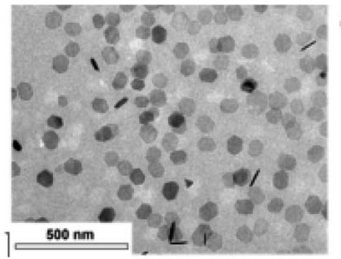

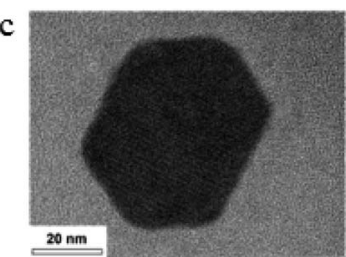

Fig. 4 (a) TEM images of the $5 \mathrm{~nm}$ GQDs distributed in methanol. (b and c) High resolution and low magnification pictures of the $70 \mathrm{~nm}$ size GQDs in methanol. Reprinted with permission. ${ }^{85}$ Copyright 2019, American Chemical Society.

recognize the elegant structures of the CQDs, the high-resolution TEM is possible to be used. CQD and GQDs are structurally distinct (Fig. 3). Fig. 3 shows the structure of CQDs and GQDs after surface modification.

CQDs, first published by Sun et al. (2006), ${ }^{17}$ have quasi spherical shape and are NPs generally less than $10 \mathrm{~nm}$ of diameter while some research observed a small number of the hollow-structured CQDs. ${ }^{81}$ On the contrary, the fluorescent GQDs that has been synthesized by Pan et al. for the first time (2010), have a crystalline structure of single or a few layered graphene. ${ }^{18}$ Although the maximum dimension published has been equal to approximately $60 \mathrm{~nm}$, but lateral dimension of the GQDs has been equal to typically a few nanometers. The synthesized GQDs form has been mostly elliptical or circular, but experts in the field addressed the synthesis of the quadrate, hexagonal, as well as triangular GQDs.

The space between layers in the two GQDs and CQDs ranged between 0.18 and $0.25 \mathrm{~nm}$ whereas the graphite inter-layer spacing ranged from 0.32 up to $0.34 \mathrm{~nm}$ or even greater interms graphite has been treated by oxidation. However, with regard to the synthetic methods, diverse deficiencies, hetero atoms as well as the functional groups have been provided, that, can change the physicochemical and structural features of the nanodots significantly. ${ }^{82,83}$

Jing et al. synthesized carbon dots using biomass as precursor via hydrothermal technique. TEM was used to assess the microstructure of CQDs and it showed that they have a uniform diameter in the range between 1.5 and $4.0 \mathrm{~nm}$. Notably, the high resolution TEM image reveals lattice fringes with $0.20 \mathrm{~nm}$ of inter-planar space that is corresponding to the facet of graphitic carbon. ${ }^{84}$

Lee et al. introduced a newly devised procedure which is used for synthesizing the size controlled single crystalline GQDs in hexagonal shapes. ${ }^{85}$ In addition, the smooth size distribution and the crystalline forms have been seen using the images obtained from TEM with a lateral size of respectively $5 \mathrm{~nm}$ (Fig. 4a) and $70 \mathrm{~nm}$ (Fig. $4 \mathrm{~b}$ and c). ${ }^{85}$

\subsection{Fourier transform infrared spectroscopy}

Some studies also illustrated that the GQDs and CQDs commonly contained oxygen, hydrogen, and carbon. Considering that carbon based QDs can be achieved by insignificant oxidation of the carbon precursor, and the CDs surface has been full of the hydroxyl, epoxy or ether, carboxylic acid or carboxyl groups, accordingly, in addition, the FTIR has been considered to be a robust device to analyze the mentioned oxygencontaining groups. Carbon based QDs mostly needed modifications for stabilization of the potential wells on the energy surface, increasing the fluorescence QY, and reducing cytotoxicity before usage of FTIR. Moreover, it is possible to use the infrared spectroscopy for characterizing the modified carbon based QDs for determining effective passivation of them. ${ }^{86,87}$

Iannazzo et al. produced GQDs with diameter of $5 \mathrm{~nm}$ using acidic oxidation of pristine MWCNT. Their infrared spectrum was recorded. The powerful wide peaks were obtained at approximately $1620 \mathrm{~cm}^{-1}$ as well as $3450 \mathrm{~cm}^{-1}$ which were related to $\mathrm{O}-\mathrm{H}$ vibrations and $\mathrm{C}=\mathrm{O}$ bonds; the peak at $1072 \mathrm{~cm}^{-1}$ was correlated with the $\mathrm{C}-\mathrm{O}$ alkoxy groups which were found in the nano-material. As demonstrated, the outputs showed the presence of several oxygenated functional groups on the surface of the GQD which resulted in their high solubility in water. $^{88}$

\subsection{UV spectroscopy}

As mentioned in the studies, GQDs and CDs have efficient applications in the photon harvesting in the short wave length area due to the $\pi-\pi^{*}$ transition of the $\mathrm{C}=\mathrm{C}$ bonds. Naturally, these dots showed stronger optical adsorption in the UV zone (260 to $320 \mathrm{~nm}$ ), while its tail extends to the visible scope. In general, the CDs had comparatively higher efficiency than GQDs in terms of absorption of the extended wave lengths. Despite CDs various GQDs demonstrate a sharp peak ranging between 270 and $390 \mathrm{~nm}$ which shows probable contribution to the $\mathrm{n}-\pi^{*}$ transition of the $\mathrm{C}=\mathrm{O}$ bonds. ${ }^{89}$ notably, surface passivation and functional groups could modify the adsorption features in the two categories.

\subsection{Photoluminescence}

One of the best features of the carbon nano-dots has been considered to the respective elegant PL features which comes from the quantum confinement impact. In fact, PL quantum yield obtained from the bare CDs declined (in general less than $10 \%$ ) which is because of its emissive traps on the surface. The QY could be enhanced dramatically via the surface passivation or modification. However, as compared in the study, the GQDS show greater QY than the bare CDs which is due to the presence of layers in their structure and better crystallinity. To date, researchers dealt with the synthesis of various CDs and GQDs using diverse methods with different PL color, in the range between UV and visible light and even close to the infrared area. However, optical properties of CDs and GQDs including the luminescence mechanism are also the most challenging issues. 
The emission peak could change with regard to the excitation wave length and it leads to an interesting while confused excitation behavior and arises debate about luminescence feature. ${ }^{89,90}$

A typical characteristic of photoluminescence in the CDs has been considered to be the obvious $\lambda_{\text {ex }}$ dependence of the emission wave length and also its intensity. Therefore, via applying the surfactant modified silica sphere as the carrier and resols as the carbon precursors, the CDs with a size range of 1.5 to $2.5 \mathrm{~nm}$ have been synthesized and later the surface passivation has been performed with PEG1500N. Therefore, final QY of the passivated CDs has been indicated as equal to $14.7 \%$. In addition, suspending the passivated CDs exhibited robust blue luminescence with excitement at $365 \mathrm{~nm}$. Moreover, the CDs had wide emission spectra, in a range between 430 and $580 \mathrm{~nm}$ so that exhibited $\lambda_{\text {ex }}$ dependent photoluminescence emission. Consequently, the vibrant and brilliant photoluminescence of the CDs could be explained due to the presence of the surface energy trap established via the surface passivation..$^{\mathbf{8 6}, 91}$

Rajender and co-workers reported synthesizing the edge controlled and strongly fluorescent GQDs with layered structure with the use of diverse solvents and explored the respective applications in the confocal imaging of the cancer cells. They revealed elegant PL emission of the GQDs in distinct liquid solutions while it has been revealed that robust PL emission of the GQDs is due to various edge locations as well the functional groups which are connected to the GQDs. They achieved a high QY equal to 32\% for PL and synthesized GQDs using DMF solvent, however it is believed that this feature is originated from the enriched edge locations and oxygen functional groups deficiencies in the GQDs. Dielectric constant of the PL from the GQDS determines its emission efficiency in the solvent medium. ${ }^{92}$

\subsection{X-ray diffraction}

Characterization of carbon based QDs and other related information including particle size, phase purity, and crystal structure is mainly carried out using XRD procedure. Moreover, this method determined the crystalline stages of the carbon based QDs. ${ }^{86}$

Chen et al. used starch as one of the novel precursors for synthesizing the GQDs in hydrothermal reaction. The XRD patterns of the GQDs showed the broader peak at nearly $24 \AA$ relative to the (002) peak that has been compatible with the JCPDS card no, 75-0444, which suggests the graphene structure has been produced using starch through the hydrothermal reaction..$^{93}$

Thambiraj et al. suggested green technique to synthesize the fluorescent CQDs using the sugarcane bagasse and exfoliation as well as the chemical oxidation. XRD characteristic peaks have been observed at $2 \theta=11.4^{\circ}, 20.6^{\circ}, 22.8^{\circ}, 42.3^{\circ}$, and $45.7^{\circ}$. Therefore, the CQDs principal peak has been obtained at $2 \theta=20.6^{\circ}$ and $42.3^{\circ}$ which is assumed to be due to the presence of the graphitic carbon. Consequently, the sharp peak has been seen at $20.6^{\circ}$ relative to the (311) plane of carbon. This diffraction pattern shows the face centered cubic crystal structure of CQDs. ${ }^{94}$

\subsection{Nuclear magnetic resonance spectrum}

As stated by a study in the field, the nuclear magnetic resonance is often used for determining the hybrid kinds of the carbon atoms in the crystalline lattices and interaction types between the carbon atoms, which also provides further structural insights about carbon base QDs. ${ }^{\mathbf{8 6}}$

Rezaii and Mahkam prepared GQDs via pyrolysing citric acid in the presence of octylamine on the catalyst. According to the researchers, the HNMR spectrum outputs obtained for the synthesized graphene in chloroform showed appropriate peaks for the modified graphene so that a sharp peak close to $1.25 \mathrm{ppm}$ and a peak about $7.5 \mathrm{ppm}$ corresponded to the $\mathrm{H}$ atom which is attached to the amine groups and bonds between octyl of a carbon group on the GQDs. ${ }^{13} \mathrm{C}$ NMR spectroscopy could differentiate $\mathrm{sp}^{2}$ and $\mathrm{sp}^{3}$ hybridized carbons and reveals the carboxyl and amide groups' signals ranging between 170 and $185 \mathrm{ppm} .{ }^{13} \mathrm{C}$ NMR confirmed the connection of alkyl groups to the graphene surface. ${ }^{95}$

Algarra et al. introduced original CQDs obtained from graphite using the green modification of the Hummers technique. Ss-NMR experiments have been performed for characterization of the CQDs chemical structure. The direct ${ }^{13} \mathrm{C}$ polarization spectrum of the CQDs just reveals the graphitic section of $\mathrm{sp}^{2}$ at a chemical shift of $128.3 \mathrm{ppm}$ in a magnetic field of $7 \mathrm{~T}$, nevertheless, in the case of recording spectrum at 14 $\mathrm{T}$, this spectrum presented further signals at 113.7 and 164.7 ppm, which are allocated to the lactol type carbon belonging to 5 and or six membered lactol ring and ester carbonyl carbon or the carboxylic acid group (-CO-O-R or $\left.-\mathrm{CO}_{2} \mathrm{H}\right)$ based on the results obtained in previous studies on graphite oxide materials. ${ }^{\mathbf{9 6}}$

\subsection{Raman spectroscopy}

Raman spectroscopy is a fast, nondestructive, and high resolution tool for the characterization of the lattice structure and the electronic, optical, and phonon properties of carbon materials, including three dimensional (3d) diamond and graphite, $2 \mathrm{~d}$ graphene, $1 \mathrm{~d}$ carbon nanotubes, and od fullerenes and carbon based QDs. ${ }^{97}$

Dervishi et al. in 2019 present a Raman spectroscopy study of bottom-up synthesized GQDs with lateral dimensions between 0.97 to $1.62 \mathrm{~nm}$, well-defined (armchair) edge type, and fully benzenoid structures. Raman spectra of GQDs reveal the presence of D and $\mathrm{G}$ bands, as well as higher order modes (2D, D + $\mathrm{G}$, and $2 \mathrm{G}$ ). The $\mathrm{D}$ and $\mathrm{G}$ band frequencies and intensity were found to increase as GQD size increases, while higher order modes $(2 \mathrm{D}, \mathrm{D}+\mathrm{G}$, and $2 \mathrm{G})$ also increased in intensity and became more well-defined. The integrated intensity ratios of $\mathrm{D}$ and $\mathrm{G}$ bands $\left(I_{\mathrm{D}} / I_{\mathrm{G}}\right)$ increase as the size of the GQDs approaches $2 \mathrm{~nm}$ and rapidly decrease for larger graphene structures. They present a quantitative comparison of $I_{\mathrm{D}} / I_{\mathrm{G}}$ ratios for the GQDS and for defects introduced into large area graphenes through ion bombardment, for which inter defect distances are comparable to the sizes of GQDs studied here. Finally, they show that Raman spectroscopy is also a good diagnostic tool for monitoring the formation of bottom-up synthesized GQDs. ${ }^{98}$ 
Kumar et al. in 2014 synthesized carbon QDs by simple heating of orange juice at $120{ }^{\circ} \mathrm{C}$ for $150 \mathrm{~min}$ without using any special apparatus or reagents. Raman spectroscopic studies have been performed for optical, electronic quantitative and qualitative analysis of the carbon QDs. Recorded Raman spectra reveals graphitic nature of carbon QDs. Sharp and intense Raman peak of G-band observed at $1578 \mathrm{~cm}^{-1}$ with respect to feeble peak of Dband at $1331 \mathrm{~cm}^{-1}$ which shows the presence of pristine carbon nanomaterials in the form of carbon QDs. A ratio of intensities of $I_{\mathrm{D}} / I_{\mathrm{G}}$ was calculated to be 0.59 which denotes the purity of the carbon QDs. ${ }^{99}$

\section{Applications of CQDs and GQDs}

The extensive properties of carbon based QDs can be used for many utilizations in diverse areas, among which drug/gene delivery, biological imaging, antibacterial and antioxidant activity and sensing applications including PL sensors, electrochemiluminescence sensors and electrochemical sensors can be mentioned. It can be stated that these applications would influence the human's life quality and has the potential to attract significant commercial interests. Many studies have been conducted in order to understand the unique features of carbon based QDs and alteration of the respective features with the use of the diverse procedures like controlling the shape and dimension, devising the composite materials, doping, and others.

\subsection{Biological applications}

4.1.1. Drug/gene delivery. Carbon dot based nanocarriers are highly interesting due to their biomedical applications, biocompatibility, biodegradability, greater efficiency, surface functionality, and physicochemical properties. Therefore, carbon based QDs are capable of bright new paths for drug and gene delivery applications. ${ }^{\mathbf{1 0 0 , 1 0 1}}$

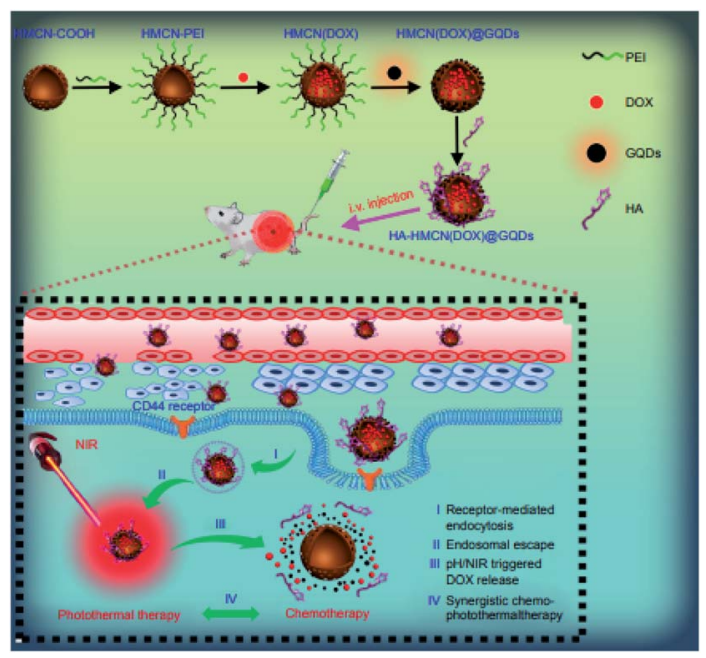

Fig. 5 A schema of the HA-HMCN(DOX) aGQDs nano-platform to target the drug delivery and synergistic chemophotothermal treatments. Reprinted with permission. ${ }^{102}$ Copyright 2018, Dovepress.
Fang et al. developed a multi functional nano platform on the basis of the hyaluronic acid (HA) modified and GQDs gated hollow mesoporous carbon NPs (HMCN) to encapsulation of an anti cancer medicine (Doxorubicin (DOX)). ${ }^{102}$ Therefore, the researchers focused on the chemophoto thermal treatment of the cancer cells with over expressed CD44 receptor. Fig. 5 represents the usage of nano-platform for the target drug delivery and simultaneous chemophoto thermal treatement. In addition, HA-HMCN(DOX)@GQDs NPs are uniformly sized as $120 \mathrm{~nm}$ while since they show cooperation with HMCN and GQDs, then may effectively form heat under NIR irradiation in order to augment the synergistic photothermal throughput. In addition, in vivo experiments demonstrated improvement in antitumor effects of the HA-HMCN(DOX)@ GQDs under the NIR irradiations. ${ }^{\mathbf{1 0 2}}$

Ghosh et al. synthesized CDs using renewable sources and conjugated them with the polyamidoamine (PAMAM) dendrimers in a variety of generations. Out of the CD-PAMAM conjugates (CDPs), the CDP3 revealed better gene protection and complexation abilities in opposition of the enzymatic digestion. However, the decreased poisonousness equal to 500 $\mu \mathrm{g} \mathrm{mL} \mathrm{m}^{-1}$ concentration, the blood compatibility as well as better efficiency of the gene transfection represent the encouraging gene delivery system to treat the triple negative breast cancer (TNBC) gene. Additionally, the CDP3 revealed high selective determination of the $\mathrm{Cu}(\mathrm{II})$ ion with a $93 \%$ fluorescence quenching efficacy and determination of the $\mathrm{Cu}$ ion at $2 \mathrm{mM}$ concentration that may be helpful for identification of metastatic stage of the triple negative breast cancer that demonstrates high levels of $\mathrm{Cu}(\mathrm{II})$ ion. ${ }^{103}$

Duan et al. stated that synthesis of the water soluble carbon dots with high number of the carboxyl groups has been performed through citric acid and urea via an easy microwave irradiation, and the as-procured CDs exhibited the average dimension equal to $2.6 \mathrm{~nm}$ with the green fluorescence emission and the fluorescence QY as $16.7 \% .{ }^{104}$ Obtained CDs showed reasonable biocompatibility and can be readily absorbed by the cells. In fact, the DOX and CDs combination via an electrostatic interaction had the medicine loading efficiency as $75.3 \mathrm{wt} \%$ (Fig. 6a). DOX can be obtained via release through the CDs-DOX a

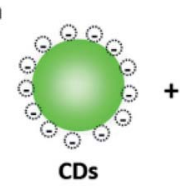

b

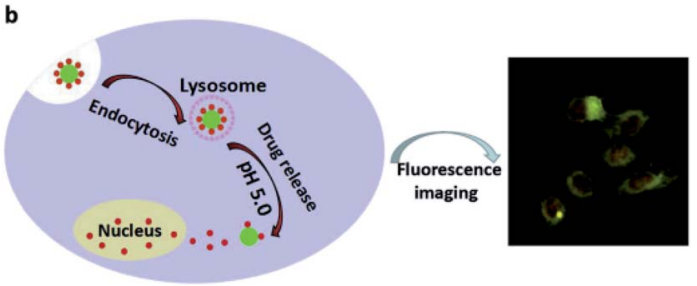

Fig. 6 A schema of the procurement process of (a) cellular uptake and (b) CDs-DOX drug delivery system. Reprinted with permission. ${ }^{104}$ Copyright 2019, Elsevier. 
system with regard to low acidic $\mathrm{pH}$ in the cancer cells that is readily entered to the nucleus (Fig. 6b). Therefore, CDs-DOX mechanism demonstrated high suppressive effects on the MGC803 cells and lower toxic impacts on the GES-1 cells. However, the CDs-DOX mechanism has advantage of being traceable for usage in the drug delivery system in the real time as an imaging agent in order to obtain images from cancer cells. ${ }^{\mathbf{1 0 4}}$

4.1.2. Bioimaging. The optical features of QDs with carbon basis are key factors of practical application of these compounds, because they release heavy fluorescence and have lower cytotoxicity and high biocompatibility that could be used in bioimaging as well as biology.

$\mathrm{Li}$ et al. suggested the usage of mitochondria as the contribute of CQD delivery system for having in vivo images. They isolated mitochondria from the cells, loaded CQD in them (Mito-CQD), and finally used it as one of the imaging agents to provide images of the cancer cells in vitro. Consequently, to provide animal imaging in vivo, they developed the nearinfrared fluorescent CQD using the modified CQD and the near-infrared Daylight 680 (Dy680) which was followed by loading CQD-Dy680 into the mitochondria (Mito-CQDDy680). ${ }^{105}$

Another study showed that lattice phosphorus (P) condensation is one of the efficient methods for adjusting the GQDs. Wang et al. reported green production of the P-GQDs using the solvothermal treatment of lecithin which obtained higher efficiency (71 wt\%). However, the final P-GQDs demonstrated appropriate emission wave length (457 to $632 \mathrm{~nm}$ ), higher quantum efficiency ( 0.54 to 0.73 ) as well as a potent to be utilized in the fluorescent bioimaging. ${ }^{106}$

Singh and coworkers synthesized GQDs applying a simple hydrothermal technique with the use of the neem root extracts as the raw materials. Therefore, the as-synthesized GQDs showed high biocompatibility, up-conversion, photostability, as well as continued full color emissions. Moreover, experiments in vitro sowed specific affinity of GQDs to lysosomes, whereas in vivo imaging applied on the zebrafish approved the preferred location of the GQDs on the yolk sac area. Thus, research reveals that such GQDs are applicable as the fluorescence imaging parameters for digestive tract. ${ }^{\mathbf{1 0 7}}$

4.1.3. Antibacterial and antioxidant activity. Kovacova et al. investigated biocompatibility and photocatalytic, anti bacterial, and structural features of the photo active hydrophobic CQDs/ poly-urethane nano composites. Then, researchers used the swell encapsulation-shrink procedure in order to produce these nanocomposites. It was found that hydrophobic CQDs/polyurethane nano composites have been very effective generators of singlet oxygen via irradiation of low power blue light. Results of the anti-bacterial experiments on the Staphyloccocus aureus and $E$. coli exhibited five logs bactericidal effects by such nano composites within sixty minutes of irradiation. Finally, the highly strong dye degradation has been achieved in 180 minutes duration of the blue light irradiation of the nano composites. ${ }^{\mathbf{1 0 8}}$

Chen and co-workers generated a newly developed anti bacterial mechanism, wherein the GQD/AgNP hybrids were used as an anti bacterial agent. Notably, these researchers have been the first to show that GQD/AgNP hybrids revealed the dual enzyme activity as the same as such effects in oxidase and peroxidase. Similar activities of the GQD/AgNP hybrids with peroxidase has been used for catalyzing the $\mathrm{H}_{2} \mathrm{O}_{2}$ decomposition into $\mathrm{OH}$ while its similar activity with oxidase as used for facilitating reactive oxygen species (ROS) formation. Considering high anti-bacterial potential of ROS, the GQD/AgNP hybrids had outstanding anti-bacterial features in opposition of the Gram-positive ( $S$. aureus), drug-resistance bacteria, and Gram-negative (E. coli). ${ }^{109}$

However, a small number of the investigations observed the anti oxidant activities of GQDs, for generation of distinct free radicals as well as their relationship with the respective compositions or $\mathrm{sp}^{2}$ hybridization structures. In a recent study by Wang et al., antioxidant activity of GQDs has been evaluated in opposition of the 1,1-diphenyl 2-picryl-hydrazyl (DPPH) with diverse oxygen fractions and volumes within all oxygen groups. Results demonstrated that the total oxygen level and the kind of the oxygen group play important roles in the GQDs anti oxidant activities. In addition, XPS has been analyzed prior to and following the reactions with DPPH, and also the Raman spectra was obtained, which all together suggested one of the key mechanisms for the GQDs free radical scavenging. This mechanism included carrying hydrogen donor from the carbonyl and hydroxy groups' surfaces, and adducting them to form at $\mathrm{sp}^{2}$ sites of the carbon network. Since the new position and chemical environment of all the surface oxygen groups were different, the $\mathrm{CQO}$ and $\mathrm{C}-\mathrm{OH}$ groups showed higher activity for free radical scavenging in comparison to the $\mathrm{C}-\mathrm{O}-\mathrm{C}$ group. ${ }^{\mathbf{1 1 0}}$

Chunduri et al. reported the green synthesis of the water soluble, monodisperse CQDs using the coconut husk, the natural biomass as one of the carbon precursors via a single phase hydrothermal carbonization. The synthesized CQDs have been in the size ranges between 1 and $8 \mathrm{~nm}$. Therefore, CQDs anti oxidant activities have been determined and $\mathrm{EC}_{50}$ has been obtained as $60 \mu \mathrm{g} \mathrm{mL}^{-1}$ with the use of the DPPH assay. ${ }^{111}$

\subsection{Sensing applications}

4.2.1. PL sensors. The usage of different methods for production of carbon based QDs caused distinct colors in photo-luminescence, from deep UV to the green, blue, red, and yellow among which the green and blue have been the commonest ones. Moreover, the photoluminescence of carbon based QDs is usually affected by diverse parameters like the shape, dimension, the excitation wave length, surface oxidation degree, concentration, surface functionalization as well as the N/S doping. ${ }^{24,89}$ Experts in the field used the special PL features of C-dots/GQDs which are established on either PL turn-off or turn-on mechanism for detecting the ions, and proteins in the solution or within the cell.

Recently, the magnetic and fluorescent nano-fiber/Fe/CDs have been synthesized by utilization of the ball milling, electrospinning, sonochemical, as well as the hydrothermal techniques. In addition, synthesis of the photoluminescence CDs has been done using the citric acid and ethylene diamine via hydrothermal technique. In the following, the Fe NPs have been added into the polymeric fibers and CDs have been coated on 
the fibers using sonochemical based hydrothermal technique. The magnetic characteristic which is observed as a result of the inner Fe NPs and photoluminescence are resulted by the surface absorbed CDs. The obtained outputs demonstrated reducing in the PL intensity via enhancement in the poisonous heavy metal ions. In fact, the magnetic nanofibers are presented as the immediate sensors to detect lead(II) and mercury(II). Moreover, the lead(II) and mercury(II) orbitals (d) receive the electron from the excited CDs. In addition, interaction between the $\mathrm{Hg}^{2+}$ (or $\mathrm{Pb}^{2+}$ ) and CDs results in forming the complex, that in consequence electron is transferred between the acceptor and donor of the electron, and fluorescence of CD is declined. ${ }^{112}$

Palomero et al. produced the fluorescent hydro gel on the basis of the S,N-codoped GQDs (S,N-GQDs) which shows activities of luminophore and sensitizer. The newly devised GQD/NC hydrogel has been used for determining the particular trichlorophenol (TCP) in the red wine and water specimens. Sensing feature is based on the capability of the graphene nanosheets for interacting with the TCP aromatic ring. Therefore, a non-covalent interaction between the aromatic analyte and the non-aggregated tiny carbon nano-sheets is reinforced probably because of stacking interaction and, based on this a highly fluorescence emission is achieved. The above feature makes utilization of the sensing behavior of PL hydro-gels against TCP possible. ${ }^{\mathbf{1 1 3}}$

Zhang et al., reported synthesis of the water soluble GQDs, emitting the up-conversion and down-conversion PL. Moreover, they devised a straightforward mixing and detection procedure for quantitatively detecting the acidic amino acids (Glu and Asp) based on the PL quenching due to the $\mathrm{Eu}^{3+}$-triggered aggregations of the GQDs as well as consequent PL recoveries because of the competition between the GQDs and acidic amino acids for $\mathrm{Eu}^{3+}$ (seen in Fig. 7). In addition, up-conversion and downconversion determination techniques could both detect sensitively and specifically the acidic amino acids. It has been found that the up-conversion state has been accompanied with the declined LOD in comparison to the down-conversion method. ${ }^{\mathbf{1 1 4}}$

4.2.2. Electrochemiluminescence sensors. Electrochemiluminescence (ECL), which is also called electrogenerated chemiluminescence, has been considered as one of the smart combinations of chemi-luminescence and electro-chemistry, and has been considered as a worthwhile determination method that is growingly applied in the analytical chemistry because of the respective simplistic setup, higher sensitivity, and label-free nature. Therefore, researchers presented some

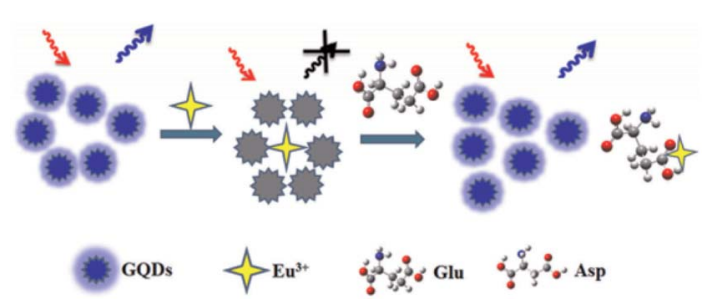

Fig. 7 The working system of the GQDs-based photo-luminescent sensors to detect Asp and Glu. Reprinted with permission. ${ }^{114}$ Copyright 2015, Elsevier. semi-conductor nano-crystals (called also semi-conductor QDs, CQDs, \& GQDs) for showing the ECL emission feature that is important to make the ECL sensors. When the light emitting samples have been formed in situ, and adjacent to the surface of the electrode, the ECL shows almost zero background that provides possibility for spatially and temporally controlling the reaction. ${ }^{\mathbf{1 1 5}}$

You et al. devised one of the methods to synthesize the CDscapped gold nano flower (CD/AuNF) nano hybrids, which contained the Au nano-particle aggregates in the centers and CDs at surfaces. Notably, the simplified self assembly of AuNFs with a number of biomolecules as well as certain ECL activity of the CDs led the observed CD/AuNF nano hybrids efficient in biosensing. As an example, the newly designed ECL aptasensor to detect thrombin (TB) has been synthesized using the CD/AuNF nano hybrids. Moreover, this aptasensor had higher selectivity for TB. In addition, the sensor had broader linear ranges of responses between $0.5 \mathrm{nM}$ and $40 \mathrm{nM}$ with a LOD equal to $0.08 \mathrm{nM}(\mathrm{S} / \mathrm{N}=3) .{ }^{116}$

Jie et al. synthesized a novel GQD with reasonable electrochemiluminescence feature. Then, they utilized it for designing a sensitive ECL biosensor in order to detect DNA with the use of the cycling amplification procedure. Moreover, the PDDA-GO complex has been utilized for immobilizing the GQDs on the electrode that enhanced the ECL signal and the electrode stability. In addition, ECL quenching of the Au NPs in GQDs has been fused with the cycling amplification procedure in order to design a sensitive ECL DNA assay technique. Finally, ECL biosensor exhibited higher sensitivity and selectivity to detect DNA. $^{117}$

Recently, it has been shown that luminol nitrogen doped GQDs (luminol-NGQDs) nano-composite has an electrochemiluminescence resonance energy transfer (ECL-RET) procedure as acceptor with luminol as a donor in the composite. Thus, such a ECL-RET effect would be helpful in the luminol-NGQDs composite to produce an anodic ECL signal with no coreactants. According to the considerable sensitization effects of the hydrogen peroxide on the luminol-NGQDS ECL signal, ECL technique was applied for sensitively detecting the hydrogen peroxide as well as the hydrogen peroxide in the water specimens. ${ }^{118}$

4.2.3. Electrochemical sensors. According to a study in the field, CQDs and GQDs have been considered as amongst the most interesting topic in studying the carbon nano-materials. They are highly considered because of the respective unique fluorescent features, and excitation wave length dependent emissions. Based on the lower toxicity, high photostability as well as chemical inertness of these compounds, experts in the field used the QDs in a variety of sciences. Generally, the CQDS and GQDs have sizes in a nanometers range. ${ }^{119}$

The zero bandgap of graphene prevents exciting and irradiating the light based on the excitation. Hence, GQDs, are formed by breaking down the graphene into a little dimension, nonzero band gap compounds and could act as the semi conductors. In fact, the GQDs have been considered to be the graphene sheets with a size $\sim 100 \mathrm{~nm}$, having the greater specific surface areas, greater surface active sites as well as 
more available edges in comparison to the graphene. ${ }^{\mathbf{1 2 0}}$ Because of the enclosure and edge effects of quantum, the GQDs had higher speed electron transfers and conductivity in addition to the respective optical features, providing them as an appropriate transducer for enhancing or controlling heterogeneous electron transfer in designing biosensors. However, the GQDs' shape, height, and edge determine their electronic and optical properties. GQDs could be achieved as the single, double-, and multi-layer substances, and develops attractive features which are helpful in generation of the electrochemical biosensors like strong chemical inertness, high photo-stability, great bio-compatibility, and lower poisonousness. GQDs also have hydroxyl, carbonyl, and amino functional groups at their edges that mediate solubility in the aqueous solutions. Moreover, they are capable of functionalizing with the organic, inorganic, or biological moiety, and also is crucial to procure the electrochemical biosensors. Finally, the mentioned groups influence the local density of the electronic state and absorption of the redox samples and also catalyze the redox reaction. ${ }^{\mathbf{1 2 1}}$

In addition, the CQDs are considered as a newly designed category of nano carbon which have a little particle size about $5 \mathrm{~nm}$, fast electron transfer, electron reservoir, and supernal conductivity features. Various functional groups on the CQDs surfaces as the interactive components makes the composites generation possible and enhances the catalytic actions of the original substances. ${ }^{\mathbf{1 2 2}}$

Hence, due to these mentioned remarkable properties, utilizing GQDs and CQDs on the modified electrode for electrochemical sensors/biosensors has been considered as an encouraging options.

The following section describes different types of CQDs and GQDs based electrochemical sensors in order to detect important neurotransmitters including tyrosine, epinephrine, norepinephrine, dopamine and serotonin, in details.

4.2.3.1. CQDs based electrochemical sensors for neurotransmitter determination. Dopamine biosensor was developed as a simple, sensitive and reliable system on the basis of CDs and chitosan (CS) composite film modified glassy carbon electrode (GCE) (CDs-CS/GCE) by Huang et al. in 2013. In addition, at optimized condition, this CDs-CS/GCE had acceptable electrochemical responses to detect DA in comparison to the GCE responses. Consequently, the DA oxidation peak current $\left(I_{\mathrm{pa}}\right)$ has been linear with its concentrations ranging between $0.1 \mathrm{mM}$ and $30.0 \mathrm{mM}$ and LOD equal to $11.2 \mathrm{nM}(3 \mathrm{~S} / \mathrm{N})$. It has been also reported that this $\mathrm{CDs}-\mathrm{CS} / \mathrm{GCE}$ has been utilized in order to detect DA contents in an injection solution of DA, resulting in successful outputs. ${ }^{123}$

Huang et al., introduced a GCE modified by the Au@CDs(Au@CDs)-chitosan (CS), Au@CDs-CS/GCE, to detect DA in another study in 2013. In comparison to the bare GCE, CDs-CS/ GCE, as well as CS/GCE, the greater catalytic activities in opposition to the DA oxidation has been exhibited by the Au@CDs-CS/GCE. In optimized condition, the DA selective determination within the linear ranges of concentration as much as $0.01-100.0 \mu \mathrm{M}$ has been observed with a LOD equal to $0.001 \mu \mathrm{M}(3 \mathrm{~S} / \mathrm{N}) .{ }^{124}$
$\mathrm{Hu}$ et al. in 2014 synthesized and described a novel DA sensor on the basis of the reduced graphene oxide (rGO)-CD composite film with the increased specificity, stability, as well as sensitivity. Normally, the rGO-CDs (GCE) had more reasonable electrochemical responses for detecting DA in comparison to the bare GCE, CDs/GCE, and GO/GCE. Moreover, the linear connection was found between the DA oxidation peak current and its concentration in the ranges between $0.01000 \mu \mathrm{M}$ and $450.0 \mu \mathrm{M}$ and LOD equal to $1.5 \mathrm{nM}$ (3S/N). Finally, the rGO-CDs/ GCE demonstrated reasonable potent to prevent the background current from the big uric acid (UA) and ascorbic acid (AA). ${ }^{125}$

In 2015 Jiang et al. developed a NCQDs which has been modified on the GCE and utilized for voltammetric detection of DA. In the mentioned study, NCQDs was synthesized via hydrothermal method. The NCQDs modified electrode demonstrated greater electrochemical responses to DA. Principally, the NCQDs displayed great electrochemical performances for detection of DA with the broader linear ranges of 0 to $1.0 \times 10^{-3} \mathrm{M}$ as well as lower LOD of $1.0 \times 10^{-9} \mathrm{M}$ at the signal-to-noise ratio equal to 3 . However, the mentioned NCQDS modified electrode had good anti interference for detecting DA in the presence of UA and or AA. Finally, it has been found that the inorganic ions did not apply any effects on the DA detection. ${ }^{\mathbf{1 2 6}}$

Jiang et al. in 2015 synthesized NCDs with the use of the microwave based approach in 10 minutes which required no solvent or catalyst and utilized it to DA sensitive electrochemically determination. The differential pulse voltammetry (DPV) has been used to calculate the LOD for DA which was equal to $1.2 \times 10^{-9} \mathrm{M}$ and had a linear dynamic range between $5.0 \times 10^{-8}$ and $8.0 \times 10^{-6} \mathrm{M} .^{127}$

In their study, Li et al. in 2015 dealt with synthesizing a strongly selective DA sensor-based on the CDs on GCE. The DA peak current enhanced on the modified electrode in 0.1 mole

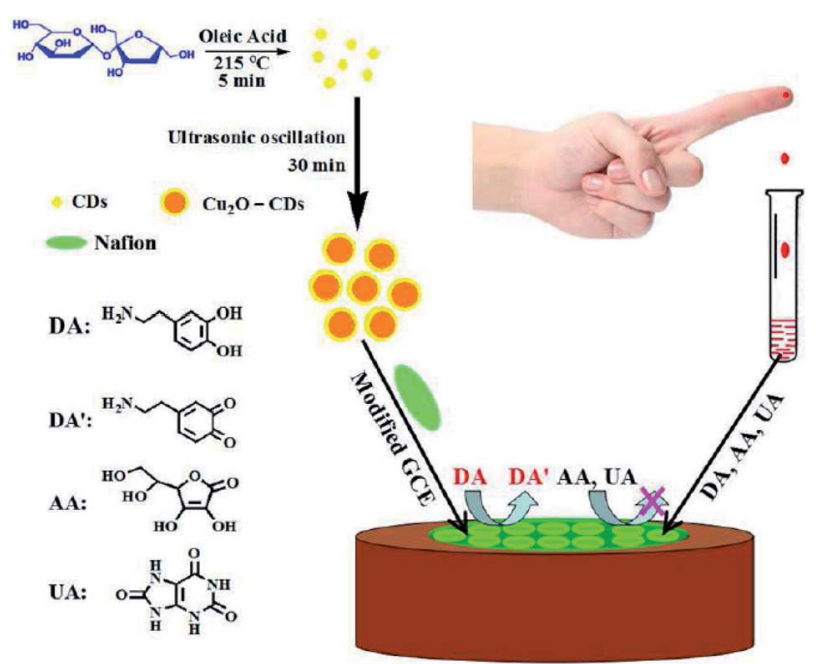

Fig. 8 A schema of the principle of electrochemical assay to detect dopamine based on cuprous the oxide-carbon dots/Nafion $\left(\mathrm{Cu}_{2} \mathrm{O}-\right.$ CDs/NF) composite film. Reprinted with permission. ${ }^{129}$ Copyright 2015 , RSC. 
per liter phosphate buffer at $\mathrm{pH}$ 6.0. Then, a calibration curve was obtained for DA between $1.5 \times 10^{-7}$ and $1.5 \times 10^{-4} \mathrm{M}$ with the LOD equal to $2.6 \times 10^{-8} \mathrm{M}$. Finally, CDs modified electrode also demonstrated the increased stability. ${ }^{\mathbf{1 2 8}}$

Huang, et al. in 2015 used a cuprous oxide carbon dots/ Nafion $\left(\mathrm{Cu}_{2} \mathrm{O}-\mathrm{CDs} / \mathrm{NF}\right.$, Fig. 8) composite film for the strongly reliable and sensitive detection DA as revealed in Fig. 8. Their bio-sensor displayed lower LOD equal to $1.1 \mathrm{nM}$ and wider linear ranges from $0.05-45.0 \mu \mathrm{M}$ as well as high selectivity and sensitivity for DA. Additionally, they used $\mathrm{Cu}_{2} \mathrm{O}-\mathrm{CDs} / \mathrm{NF} / \mathrm{GCE}$ for detecting DA in the human serum and acceptable outputs were obtained and exhibited a high activity for over 2 months. ${ }^{\mathbf{1 2 9}}$

The study conducted by Wang et al. (2016) synthesized a sensitive electrochemical sensor on the basis of the flower like NiAl with layered structure and double hydroxides (NiAl-LDHs)/ CDs modified GCE and utilized it to determine acetylcholine (ACh). Notably, this flower-like NiAl-LDHs had a crosslinking nano sheet structure as well as higher specific surface areas and was produced using hydrothermal method. In the following, the CDs with negative surface charges have been decorated on the NiAl-LDHs with the positive charged brucite like layers for obtaining the NiAl-LDH/CD composites. In comparison to the NiAl-LDHs, the NiAl-LDH/CD composites demonstrated high electroconductivity and electrocatalytic performances for ACh oxidation. Moreover, NiAl-LDH/CD composites modified GCE possessed wider linear response in the range from 5.0-6885.0 $\mu \mathrm{M}$, higher sensitivity equal to $133.20 \pm 0.03 \mathrm{~mA} \mathrm{M}^{-1} \mathrm{~cm}^{-2}$ and low LOD equal to $1.7 \mu \mathrm{M}$. In addition, biosensor also revealed high durability, the extended stability as well as very good selectivity to detect ACh. ${ }^{\mathbf{1 3 0}}$

As stated in the research, concurrent determination of DA and epinephrine (EP) has been conducted by Canevari et al. (2016), in which CQDs have been synthesized using chronoamperometric method. The C-dots have been utilized immediately after production on the GCE surface, and showed high electrochemical responses in the process of concurrent detection of EP and DA. As a result, LODs for 2 analytes respectively have been $4.6 \mathrm{nM}$ and $6.1 \mathrm{nM}^{131}$

Zhuang et al. in 2016 introduced a DA sensor on the basis of a GCE modified with the composite film which was consisted of the C-dots and the graphene functionalized with an ionic liquid (IL). Then, carboxy groups have been used to functionalize these C-dots whose negative charges promoted the electro static attractions to the protonated amino groups in DA. Notably, the presence of the imidazole cationic group in IL facilitated its interaction with the C-dots as well as DA through electro-static interaction and $\pi$-stacking force. Normally, the modified GCE demonstrates the greater electrochemical responses to DA in comparison with the bare GCE or GCE modified with the ILgraphene or C-dots. Thus, the best oxidation current has been measured at the potential of $0.22 \mathrm{~V}$ versus $\mathrm{Ag} / \mathrm{AgCl}$ which had a linear relationship with the DA concentration in the ranges between 0.1 and $600.0 \mu \mathrm{M}$ with the LOD equal to $30.0 \mathrm{nM}$ at the signal to noise ratio equal to $3 .^{\mathbf{1 3 2}}$

In 2017 , Chen et al. introduced a poly ( $\beta$-cyclodextrin) $(\beta-\mathrm{CD}) /$ CQDs composite as a new sensory platform for simultaneously determining DA, UA, and tryptophan (Trp). The electrochemical response of the as-procured electrode has been examined by cyclic voltammetry (CV), electrochemical impedance spectroscopy (EIS), and DPV. The linear responses for DA have been specified in the concentration ranges from 4.0-220.0 $\mu \mathrm{M}$, with limitation of detection of $0.14 \mu \mathrm{M}(\mathrm{S} / \mathrm{N}=3)$ and quantification limit of $0.45 \mu \mathrm{M}^{133}$

Fang et al. in 2017, introduced that CDs decorated on the graphene oxide (GO) nano sheets which have been electrochemically reduced and then deposited over the carbon fiber (CF) in order to produce micro electrodes ( $\mathrm{rGO}+\mathrm{CD} / \mathrm{CF}$ ) for strongly selective and sensitive detection of DA in the presence of UA and AA. Then, the obtained outputs revealed that modifying the surface increased electrocatalytic activities of the carbon fiber micro electrode considerably. Therefore, $\mathrm{rGO}+\mathrm{CD} /$ CF micro electrode demonstrated the linear DA determination concentrations in the ranges between 0.1 and $100.0 \mu \mathrm{M}$ and LOD equal to $0.02 \mu \mathrm{M}$. Thus, the micro electrode sensitivity against DA has been equal to $6.5 \mathrm{nA} \mathrm{mM}^{-1} .^{134}$

Devi et al. in 2018 synthesized CQDs via green electrochemical method. Next, CQDs used for the modification of the GCE and screen-printed carbon electrode (SPCE) for studying the respective electro-chemical and electrocatalytic features. It has been found that the SPCE/CQD and GCE/CQD had greater electrocatalytic activities in order to detect DA oxidation in the phosphate buffered saline (PBS) ( $\mathrm{pH}$ equal to 7.4). Then, the effect of the rate of the scan on the DA oxidation has been examined from 10 to $150 \mathrm{mV} \mathrm{s}^{-1}$. Moreover, calibration curve has been recorded for DA between 1.0 and $7.0 \mu \mathrm{M}$ with the use of a SPCE/CQD and LOD has been obtained equal to $0.099 \mu \mathrm{M}$. Finally, the reported electrocatalytic activities of CQD has been based on their functional groups with negative charge that attracted DA with positive charges in the 0.1 M PBS solution. ${ }^{135}$

Algarra et al. in 2018 reported a DA sensor wherein a CQDs modified GCE has been used to synthesize it. According to this study, the CQDs have been assembled on the GCE via adsorbing due to the larger numbers of the carboxy groups on their surfaces which certifies efficient absorption. Therefore, this electrode has been substantially utilized in order to assay the medicine in the human plasma specimens. Moreover, this modified GCE showed ten times higher sensitivity compared to the bare GCE. Finally, the decreased LOD equals $2.7 \mu \mathrm{M}$ for DA. ${ }^{136}$

Moreover, Yola et al. (2019) developed an electrochemical sensor on the basis of the molecularly imprinted polymer/ graphitic carbon nitride/N-doped carbon dots composite (MIP/g- $\mathrm{C}_{3} \mathrm{~N}_{4} / \mathrm{NCDS}$ ) and has been applied to detect EP in the urine samples. In the following, EP imprinted voltammetric sensor on the graphitic carbon nitride/NCDS has been synthesized in the presence of $100.0 \mathrm{mM}$ pyrrole consisting of $25.0 \mathrm{mM}$ EP through CV. In fact, the EP imprinted electrode demonstrated higher sensitivity to recognize EP at the broad linear ranges from $1.0 \times 10^{-12}$ to $1.0 \times 10^{-9} \mathrm{M}$ and LOD equal to $3.0 \times 10^{-13} \mathrm{M}^{137}$

The usage of carbon QDs based on modified biosensors and sensors have been surveyed in Table 1 . This summarized data present the progression and individual trends mentioned previously. 
Table 1 Selected applications of carbon QDs in modified biosensors and sensors

\begin{tabular}{|c|c|c|c|c|c|c|}
\hline Sensor & $\begin{array}{l}\text { Electrochemical } \\
\text { method }\end{array}$ & Analyte & Detection limit & Linear range & Sensitivity & Ref. \\
\hline $\mathrm{CDS}-\mathrm{CS} / \mathrm{GCE}$ & DPV & Dopamine & $11.2 \mathrm{nM}$ & $0.1-30.0 \mu \mathrm{M}$ & $-0.0212 \mu \mathrm{A} \mu \mathrm{M}^{-1}$ & 123 \\
\hline Au@CDs-CS/GCE & DPV & Dopamine & $0.001 \mu \mathrm{M}$ & $0.01-100.0 \mu \mathrm{M}$ & $-0.0353 \mu \mathrm{A} \mu \mathrm{M}^{-1}$ & 124 \\
\hline rGO-CDs/GCE & DPV & Dopamine & $1.5 \mathrm{nM}$ & $0.01000-450.0 \mu \mathrm{M}$ & $-0.00274 \mu \mathrm{A} \mu \mathrm{M}^{-1}$ & 125 \\
\hline NCQDs/GCE & DPV & Dopamine & $1.0 \times 10^{-9} \mathrm{M}$ & $0-1 \times 10^{-3} \mathrm{M}$ & $42.85 \mu \mathrm{A} \mu \mathrm{M}^{-1}$ & 126 \\
\hline NCDs/GCE & DPV & Dopamine & $1.2 \times 10^{-9} \mathrm{M}$ & $\begin{array}{l}5.0 \times 10^{-8} \text { to } \\
8.0 \times 10^{-6} \mathrm{M}\end{array}$ & $0.563 \mu \mathrm{A} \mu \mathrm{M}^{-1}$ & 127 \\
\hline $\begin{array}{l}\text { Carbon dot } \\
\text { modified glassy } \\
\text { carbon electrode }\end{array}$ & DPV & Dopamine & $2.6 \times 10^{-8} \mathrm{M}$ & $\begin{array}{l}1.5 \times 10^{-7} \text { to } \\
1.5 \times 10^{-4} \mathrm{M}\end{array}$ & $3.74 \mu \mathrm{A} \mu \mathrm{M}^{-1}$ & 128 \\
\hline $\mathrm{Cu}_{2} \mathrm{O}-\mathrm{CDs} / \mathrm{NF}$ & DPV & Dopamine & $1.1 \mathrm{nM}$ & $0.05-45.0 \mu \mathrm{M}$ & $0.0773 \mu \mathrm{A} \mu \mathrm{M}^{-1}$ & 129 \\
\hline $\mathrm{NiAl}-\mathrm{LDH} / \mathrm{CD} / \mathrm{GCE}$ & Amperometry & Acetylcholine & $1.7 \mu \mathrm{M}$ & $5-6885 \mu \mathrm{M}$ & $133.20 \pm 0.03 \mathrm{~mA} \mathrm{M}^{-1} \mathrm{~cm}^{-2}$ & 130 \\
\hline \multirow[t]{2}{*}{ GCE/C-dots } & DPV & Dopamine & $4.6 \mathrm{nM}$ & $\begin{array}{l}0.05 \times 10^{-6} \text { to } \\
2.0 \times 10^{-6} \mathrm{M}\end{array}$ & - & 131 \\
\hline & & Epinephrine & $6.1 \mathrm{nM}$ & $\begin{array}{l}0.05 \times 10^{-6} \text { to } \\
2.0 \times 10^{-6} \mathrm{M}\end{array}$ & 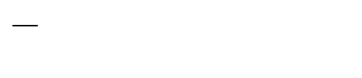 & \\
\hline C-dots/IL-graphene/GCE & DPV & Dopamine & $30 \mathrm{nM}$ & 0.1 to $600 \mu \mathrm{M}$ & $-(0.4706 \pm 0.0001) \mu \mathrm{A} \mu \mathrm{M}^{-1}$ & 132 \\
\hline$\beta-\mathrm{CD} / \mathrm{CQDS} / \mathrm{GCE}$ & DPV & Dopamine & $0.14 \mu \mathrm{M}$ & $4-220 \mu \mathrm{M}$ & $(0.288 \pm 0.006) \mu \mathrm{A} \mu \mathrm{M}^{-1}$ & 133 \\
\hline $\begin{array}{l}\mathrm{rGO}+\mathrm{CD} / \mathrm{CF} \\
\text { microelectrode }\end{array}$ & DPV & Dopamine & $0.02 \mu \mathrm{M}$ & $0.1-100 \mu \mathrm{M}$ & $0.0065 \mu \mathrm{A} \mu \mathrm{M}^{-1}$ & 134 \\
\hline CQD-SPCE & CV & Dopamine & $0.099 \mu \mathrm{M}$ & $1-7 \mu \mathrm{M}$ & $0.096 \mu \mathrm{A} \mu \mathrm{M}^{-1}$ & 135 \\
\hline GCE modified with CQDs & LSV & Dopamine & $2.7 \mu \mathrm{M}$ & $0.19-11.81 \mu \mathrm{M}$ & $9.3 \mathrm{~mA} \mu \mathrm{M}^{-1}$ & 136 \\
\hline $\begin{array}{l}\mathrm{MIP} / \mathrm{g}-\mathrm{C}_{3} \mathrm{~N}_{4} / \\
\operatorname{NCDS}(8.0 \%) / \mathrm{GCE}\end{array}$ & DPV & Epinephrine & $3.0 \times 10^{-13} \mathrm{M}$ & $\begin{array}{l}1.0 \times 10^{-12} \text { to } \\
1.0 \times 10^{-9} \mathrm{M}\end{array}$ & $10.02 \mu \mathrm{A} \mu \mathrm{M}^{-1}$ & 137 \\
\hline
\end{tabular}

4.2.3.2. GQDs based electrochemical (bio) sensors for neurotransmitter determination. Pang et al. in 2016 developed an electrochemical sensor using GQDs and the Nafion composite. The GQDs have been synthesized via a hydrothermal strategy for breaking down graphene sheets into the GQDs. Therefore, the GQDs-Nafion composite demonstrated an appropriate linear range between $0.005 \mu \mathrm{M}$ and $100 \mu \mathrm{M}$ as well as LOD equal to $0.45 \mathrm{nM}$ for detecting DA. Finally, the suggested electrochemical sensor showed high stability and selectivity. ${ }^{\mathbf{1 3 8}}$

In 2016, Chen et al. proposed an applicable procedure for preparing the GQDs with the use of the commercial polyacrylonitrile (PAN) on the basis of the carbon fibers as the raw materials via the chemical oxidation and the exfoliation procedure. Therefore, the as-synthesized GQDs also revealed comparatively higher catalytic activities in oxidation of DA and have the potential as the sensor for detecting DA in the concentration range of 3.0-100.0 $\mu \mathrm{M}^{139}$

L-Tyrosine (L-Tyr) concentration was detected using a renewable carbon composite electrode which was modified by GQD$\mathrm{RuCl}_{3}$ in a study conducted by Habibi and Heidari (2016). Therefore, GQD has been produced via pyrolysis of the citric acid and consequently utilized in a nanocomposite carbon electrode. The synergistic interpolation of the $\mathrm{RuCl}_{3}$ with GQD enhances sensor sensitivity. Therefore, amounts of sensitivity and LOD have been determined to be $90 \mathrm{nA} \mu \mathrm{M}^{-1}$ and0.23 $\mu \mathrm{M}$. Moreover, produced sensor displayed a lengthy stability and reasonable selectivity to detect L-Tyr. ${ }^{\mathbf{1 4 0}}$

Hasanzadeh et al. in 2016, reported synthesis of a novel nano composite, the $\mathrm{Fe}_{3} \mathrm{O}_{4}$ MNP-GQDs to sense a number of amino acids. Moreover, the as-synthesized $\mathrm{Fe}_{3} \mathrm{O}_{4}$ MNPs-GQDs and
GQDs has been electrodeposited on the GCE through CV approach for the first time, in the potent ranges between -1.0 and $1.0 \mathrm{~V}$. however results indicated that $\mathrm{Fe}_{3} \mathrm{O}_{4}$ MNPsGQDs film is capable of accelerating the rate of the electron transfer of the chosen amino acids and had reasonable electrocatalytic activities for the redox reaction of $\mathrm{L}$-cysteine, L-aspartic acid, L-Tyr, and L-phenylalanine. Hence, $\mathrm{Fe}_{3} \mathrm{O}_{4}$ MNPs-GQDs has been considered as one of the appropriate mediators for transfer electrons between the amino acids and involved electrode so that facilitated electrochemical re-generation after the exchange of electron with the amino acids. This feature can have relationship with great features of $\mathrm{Fe}_{3} \mathrm{O}_{4}$ MNPs-GQDs like higher specific surface areas and the electrical conductivity. They determined L-Tyr in the linear ranges from $0.09-230.0 \mu \mathrm{M} .{ }^{\mathbf{1 4 1}}$

Yola et al. in 2016 developed the voltammetric sensor for development of concurrent detection of AA, DA, tryptophan, and UA on rAu-PtNPs/GQDs/GCE. The obtained sensor showed high sensitivity for target molecules with LOD equal to $3.0 \times 10^{-10} \mathrm{M}$. In addition, it has been possible to be reused, and also was selective and stable. A high percentage of recovery shows that produced sensor can be used for quantification without interference. ${ }^{\mathbf{1 4 2}}$

GQDs immobilized on GCE through covalent bond via self assembled method ((GQDs- $\left.\left.\mathrm{NHCH}_{2} \mathrm{CH}_{2} \mathrm{NH}\right) / \mathrm{GCE}\right)$ was synthesized by Li et al. (2016). Then, these GQDs have been procured via carbonizing citric acid. Consequently, (GQDs- $\mathrm{NHCH}_{2} \mathrm{CH}_{2}$ $\mathrm{NH} / \mathrm{GCE}$ revealed the linear ranges between 1.0 and $150.0 \mu \mathrm{M}$ and the LOD equal to $0.115 \mu \mathrm{M}(\mathrm{S} / \mathrm{N}=3)$ in order to detect DA, which can be considered as confirmation for high sensitivity of modified electrode for detection of DA. ${ }^{143}$ 
Ruiyi et al. in 2017 developed a strategy for synthesizing the histidine based functionalized GQD-graphene micro aerogel (His-GQD-GMA) with the use of the Pickering emulsion in the soft template method. Consequently, the sensor developed on the basis of the His-GQD-GMA demonstrated ultra sensitive electrochemical responses to DA. Moreover, its differential pulse in the voltammetric signal experienced a linear increase with an enhancement in DA concentration in ranges between $1.0 \times 10^{-9} \mathrm{M}$ up and $8.0 \times 10^{-5} \mathrm{M}$ with LOD equal to $2.9 \times 10^{-10} \mathrm{M}$ at the $\mathrm{S} / \mathrm{N}$ ratio equal to $3 .^{\mathbf{1 4 4}}$

Highly selective DA electrochemical sensor with the basis of modifying a nano structured CSPE and a chitosan/nitrogen doped GQDs nano composite was introduced by Ben Aoun in 2017. The as-procured CS/N, GQDs@SPCE demonstrated higher sensitivity; that is, ca. $418 \mu \mathrm{A} \mathrm{mM} \mathrm{cm}{ }^{-2}$, wider linear ranges from 1 to $100 \mu \mathrm{M}$ and 100 to $200 \mu \mathrm{M}$ and also very lower LOD $(\mathrm{LOD}=0.145 \mu \mathrm{M})$. Therefore, utility of the synthesized sensor was tested using real sample by detecting DA in THE humans' urines. ${ }^{\mathbf{1 4 5}}$

Baluta et al. introduced an electro-chemical sensing procedure to detect EP on the bases of the GQDs and laccase modified GCEs in 2018. After optimization, performance analysis results demonstrated high degree of sensitivity as much as $-2.9 \mathrm{~mA} \mathrm{mM}^{-1} \mathrm{~cm}^{-2}$, selectivity in the broader linear ranges $\left(1.0-120.0 \times 10^{-6} \mathrm{M}\right)$ and LOD equal to $83 \mathrm{nM}$. In addition, this technique has been substantially utilized for EP detection in the labeled pharmacological specimens. ${ }^{146}$

Shadjou et al. used the GQD- $\beta$-cyclodextrin modified GCE to detect L-Tyr as a novel nano sensor in 2018. The obtained the transfer coefficient $(\alpha)$ and electron transfer rate constant $\left(k_{\mathrm{s}}\right)$ were detected by $\mathrm{CV}$ while obtained results have been nearly equaled 8.0 $\mathrm{s}^{-1}$ and 0.7 , respectively. The modified electrode displayed higher catalytic activities to oxidize $\mathrm{L}_{\text {-Tyr }}$ in the broader linear ranges from 0.1-1.5 $\mu \mathrm{M}$ at positive potential in buffer solution. ${ }^{\mathbf{1 4 7}}$

Yola et al. used molecularly imprinted method for devising a novel electro-chemical sensor to detect serotonin (SER) in the urine specimens in 2018. Therefore, this sensor has been synthesized on the basis of the GQDs with incorporation of 2-D hexagonal boron nitride (2D-hBN) nano-sheets (GQDs/2D-hBN)

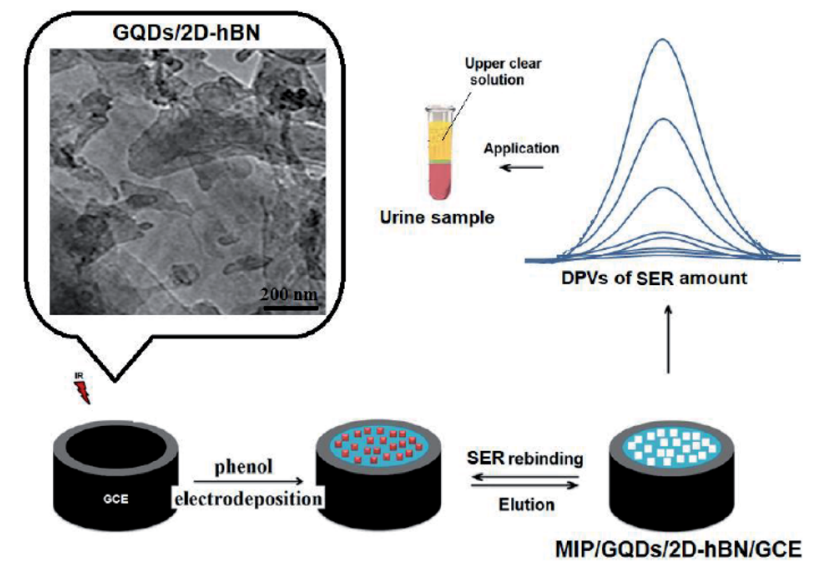

Fig. 9 MIP/GQDs/2D-hBN/GCE process. Reprinted with permission. ${ }^{148}$ Copyright 2018, Elsevier. as it has been shown in Fig. 9. $1.0 \times 10^{-12}$ to $1.0 \times 10^{-8} \mathrm{M}$ and $2.0 \times 10^{-13} \mathrm{M}$ have been obtained as the linearity range and LOD, respectively. Additionally, the devised sensor had stability and selectivity. Furthermore, the MIP/GQDs/2D-hBN/GCE (Fig. 9) has been applied for analysis of the essential neurotransmitters with no interferences. ${ }^{148}$

Zheng et al., synthesized a GCE which has been modified by the GQDs (GQD/GCE) with the aim of determination of DA in 2018. The electrochemical behaviors of the modified electrode for the catalytic oxidation of DA at $\mathrm{pH}$ equals to 7.0 created using the phosphate buffer solution (PBS) has been examined by CV and DPV. $0.4-100.0 \mu \mathrm{M}$ and $0.05 \mu \mathrm{M}$ were obtained as the amounts of linearity range and detection limit. ${ }^{149}$

Beitollahi et al. introduced graphene QDs modified screen printed electrode (GQD/SPE) as a sensor aimed to determine DA in the presence of Tyr in 2018. However, the use of the GQD/SPE led to separating in the oxidation peak as much as $435 \mathrm{mV}$ for DA and Tyr. Moreover, calibration curves showed ranges from 0.1-1000.0 to 1.0-900.0 $\mu \mathrm{M}$ for DA and Tyr, respectively. In addition, LODs $(\mathrm{S} / \mathrm{N}=3)$ have been measured respectively as 0.5 and 0.05 for Tyr and DA. Furthermore, diffusion coefficients with the use of the chronoamperometry at the modified electrode surface have been obtained respectively as $6.4 \times 10^{-5} \mathrm{~cm}^{2}$ $\mathrm{s}^{-1}$ and $9.0 \times 10^{-5} \mathrm{~cm}^{2} \mathrm{~s}^{-1}$ for Tyr and DA. ${ }^{150}$

In 2017, Tashkhourian et al. also combined GQDs and chitosan as a stabilizer in a carbon paste electrode (CPE) with an
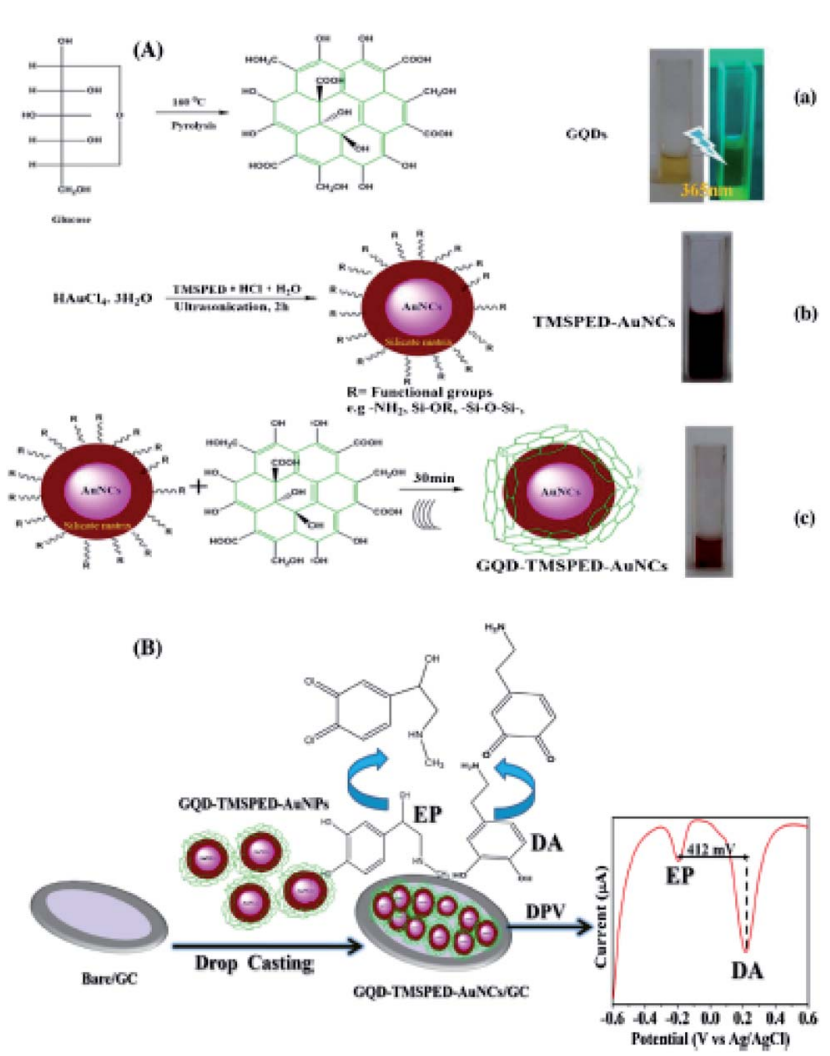

Fig. 10 A schema of the procurement process of (A) GQD-TMSPEDAuNCs nano-composites and (B) electro-chemically concurrent determination EP and DA. Reprinted with permission. ${ }^{153}$ Copyright 2019, Springer. 
Table 2 Selected applications of graphene QDs in modified sensors and biosensors

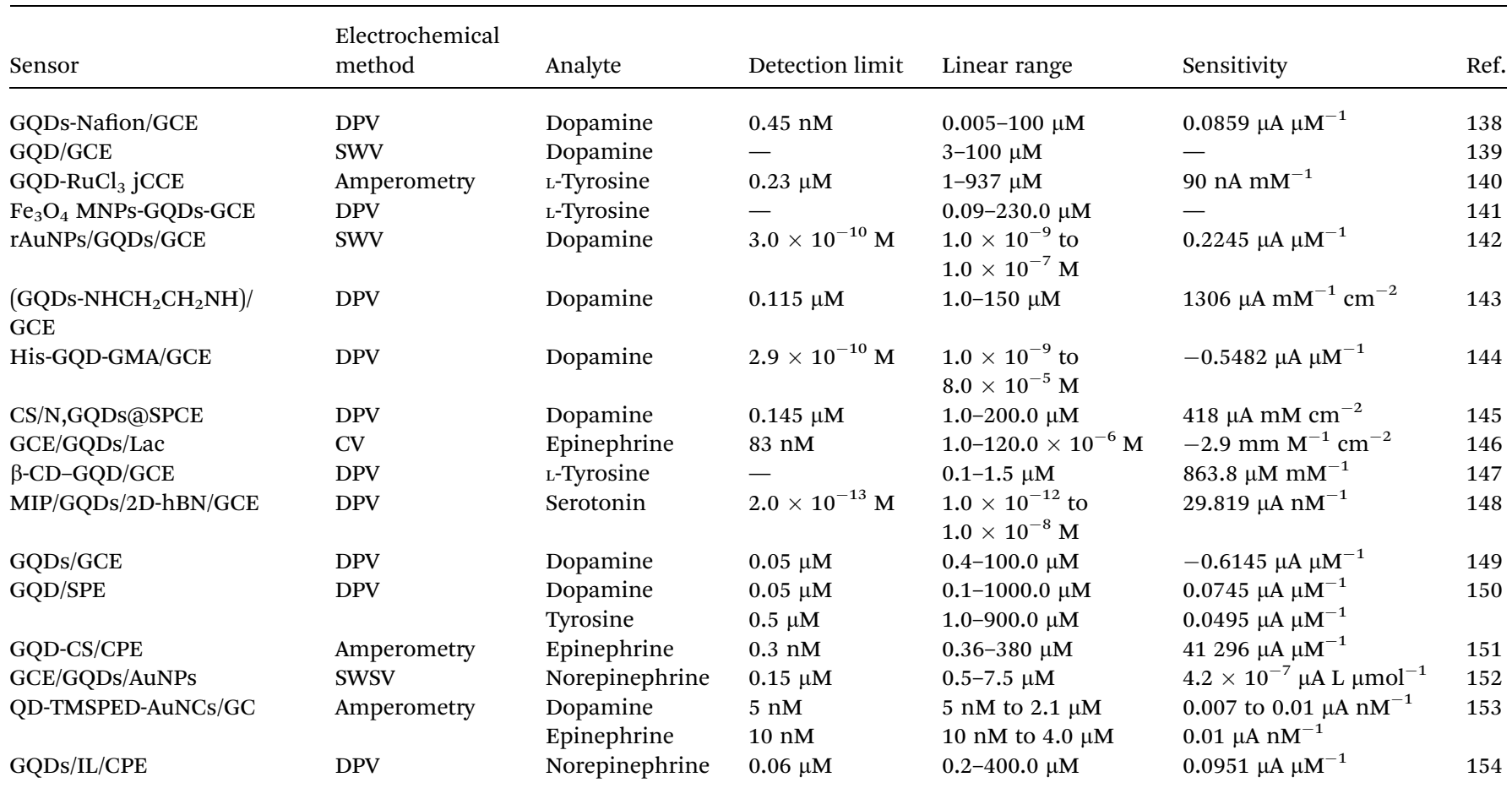

aim of voltammetric determination of EP. The EIS and CV demonstrated fast electron transfer for the prepared GQD-CSCPE compared to the bare electrode and an appropriate electrocatalytic activity to electro oxidizes EP. In the optimal experimental condition, this modified electrode showed the linear connection between EP concentration and peak current in the wider ranges between 0.36 and $380 \mu \mathrm{M}$ with LOD equal to $0.3 \mathrm{nM}\left(3 S_{\mathrm{b}} / m\right) .^{151}$

Fajardo et al. introduced a GCE modified with the GQDs and gold NPs (GCE/GQDs/AuNPs) has been devised in order to determine norepinephrine (NE) with the use of the square wave stripping voltammetry in 2019. The conditions were optimized for detecting NE with the GCE/GQDs/AuNPs. However, the linear ranges have been reported from 0.5-7.5 $\mu \mathrm{M}$ with the LOD equal to $0.15 \mu \mathrm{M}$. Suggested approach has been validated using spiked samples through which showed good precision and accuracy. Successful results were obtained for usage of GCE/ GQDs/AuNPs in the drug procurement (NE ampoules) and in the rat brain tissue. ${ }^{152}$

Vinoth et al. developed a cost-effective and environmentally friendly sonochemical approach to procure the GQDs which smoothly dispersed on the silicate matrix (TMSPED) which was capped by the gold nano crystals (AuNCs). The GQD-TMSPEDAuNCs placed on a GCE for determining EP and DA simultaneously as it is depicted in Fig. 10. The response showed linear in the concentration ranging from $5 \mathrm{nM}$ to $2.1 \mu \mathrm{M}$ (DA) to $10 \mathrm{nM}$ to $4.0 \mu \mathrm{M}(\mathrm{EP})$ with LOD equal to 5 and $10 \mathrm{nM} .^{153}$

Mohammadzadeh Jahani et al. synthesized a powerful voltammetric sensor for simultaneous analysis of NE and acetylcholine in 2020. The sensor was produced via modification of carbon paste electrode with graphene QDs/ionic liquid (GQDs/ $\mathrm{IL} / \mathrm{CPE}$ ). The GQDS/IL/CPE revealed two separated oxidation peaks for simultaneous analysis of NE and acetylcholine with $\Delta E \sim 390 \mathrm{mV}$. The calibration curve was obtained by DPV in optimized conditions, and demonstrated the linear ranges between $0.2-400.0 \mu \mathrm{M}$ for $\mathrm{NE}$ (LOD equal to $0.06 \mu \mathrm{M}$ ). The produced sensor has been substantially utilized to determine the analytes in the real specimens. ${ }^{154}$

The application of graphene QDs based on modified sensors and biosensors have been summarized in Table 2 which presents progression and single trends mentioned previously.

\section{Conclusions}

Hereby, we conducted a comprehensive review study on new carbon based QDs which includes synthesis, and characterization methods, and also covers a wider range of utilizations in the biological and sensing areas. We believe that either synthesis method or application of carbon based QDs covers a wider domain, extending further on the outlined information presented in the present review.

We focused on application of these compounds in electrochemical sensors with the aim of important neurotransmitters detection. Considering that in a complicated context like the brain, analyzing the chosen neurotransmitters which provide at the decreased concentration in comparison to a majority of the other molecular samples, measuring these molecules exposes several challenges. Electrochemistry has also its own limitations, while it also has significant advantages compared to the remaining procedures. This would provide a ground for 
detection of neurotransmitter and monitoring them via the wider range of the procedures with regard to the required information. Nonetheless, probably the most important feature has been the continuous and rapid improvements in the sensor production and development, which lead to facilitate in vivo chemistry in the commonly smaller settings. In last years, most of the studies were concentrated on synthesis of the novel sensing probe substances in order to enhance the sensor functions like selectivity, sensitivity as well as biocompatibility. Among the synthesized nano materials, carbon based QDs (CQDs and GQDs) had widespread utilization in constructing the surface of the sensor. However, such carbon based QDs also suggest great enhancement in the sensor sensitivity, selectivity, and stability. Additionally, direct electron transfer procedure in the electroactive neurotransmitters has been further considered in comparison with the reported detection procedures.

\section{Abbreviations}

$\begin{array}{ll}\text { GQDs } & \text { Graphene quantum dots } \\ \text { CQDs } & \text { Carbon quantum dots } \\ \text { NTs } & \text { Neurotransmitters } \\ \text { CNS } & \text { Central nervous system } \\ \text { SIDS } & \text { Sudden infant death syndrome } \\ \text { MS } & \text { Mass spectrometry } \\ \text { CE } & \text { Capillary electrophoresis } \\ \text { LOD } & \text { Limits of detection } \\ \text { NPs } & \text { Nanoparticles } \\ \text { QDs } & \text { Quantum dots } \\ \text { OFDs } & \text { Organic fluorescent dyes } \\ \text { C-QDs } & \text { Carbon quantum dots } \\ \text { PEG } & \text { Poly-ethylene glycol } \\ \text { N } & \\ \text { PPEI-EI } & \text { Poly(propionyl ethylene eimine-coethyleneimine) } \\ \text { SWCNTs } & \text { Single-walled carbon nanotubes } \\ \text { N-CDs } & \text { Nitrogen-carbon dots } \\ \text { WCDs } & \text { White fluorescent carbon dots } \\ \text { PL } & \text { Photoluminescence } \\ \text { abrEDTA } & \text { Ethylene diamine tetra-acetic acid; NCQDs nitrogen- } \\ & \text { doped CQDs } \\ \text { SF-CQD } & \text { Silk fibroin-based CQD } \\ \text { PES } & \text { Poly-ethersulfone } \\ \text { mSiO } & \text { Mesoporous silica } \\ \text { TMB } & \text { 1,3,5-Trimethylbenzene } \\ \text { DAB } & \text { Diaminebenzene } \\ \text { HTC } & \text { Hydrothermal carbonization } \\ \text { DA } & \text { Dopamine } \\ \text { BCP } & \text { Block co-polymers } \\ \text { CVD } & \text { Chemical vapor deposition } \\ \text { NaBiO } & \text { Sodium bismuthate } \\ \text { HF } & \text { Hydrofluoric acid } \\ \text { TATB } & \text { 1,3,5-Triamino-2,4,6-trinitrobenzene } \\ \text { TEM } & \text { Transmission electron microscope } \\ \text { NMR } & \text { Nuclear magnetic resonance } \\ \text { FTIR } & \text { Fourier transform infrared spectroscopy } \\ \text { XRD } & \text { X-ray diffraction } \\ \text { DOX } & \text { Doxorubicin } \\ & \end{array}$

PAMAM Polyamidoamine

TNBC Triple negative breast cancer

ROS Reactive oxygen species

DPPH 1,1-Diphenyl 2-picryl-hydrazyl

ECL Electrochemiluminescence

TB Thrombin

ECL-RET Electrochemiluminescence resonance energy transfer

CS Chitosan

GCE Glassy carbon electrode

rGO Reduced graphene oxide

UA Uric acid

AA Ascorbic acid

DPV Differential pulse voltammetry

Ach Acetylcholine

EP Epinephrine

IL Ionic liquid

SPCE Screen-printed carbon electrode

PBS Phosphate buffered saline

PAN Poly-acrylonitrile

L-Tyr L-Tyrosine

CV Cyclic voltammetry

EIS Electrochemical impedance spectroscopy

SER Serotonin

CPE Carbon paste electrode

NE Norepinephrine

MIP Molecularly imprinted polymer

\section{Conflicts of interest}

There are no conflicts to declare.

\section{References}

1 N. F. Atta, Y. M. Ahmed and A. Galal, Electrochemical Determination of neurotransmitters at crown ether modified carbon nanotube composite: application for sub-nano-sensing of serotonin in human serum, Electroanalysis, 2018, 31, 1204.

2 M. Yamada, A. Inanobe and Y. G. Kurachi, protein regulation of potassium ion channels, Pharmacol. Rev., 1998, 50, 723.

3 P. Baraneedharan, Graphene based biosensors for detection of neurotransmitters, J. Nanomed. Res., 2016, 3, 00066.

4 N. F. Atta, A. Galal and S. M. Azab, Electrochemical determination of neurotransmitters using gold nanoparticles on nafion/carbon paste modified electrode, J. Electrochem. Soc., 2012, 159, H765.

5 D. M. Tomkins and E. M. Sellers, Addiction and the brain: the role of neurotransmitters in the cause and treatment of drug dependence, Can. Med. Assoc. J., 2001, 164, 817.

6 A. L. Dunn and R. K. Dishman, 2 Exercise and the Neurobiology of Depression, Exerc. Sport Sci. Rev., 1991, 19, 41-98. 
7 M. Perry, Q. Li and R. T. Kennedy, Review of recent advances in analytical techniques for the determination of neurotransmitters, Anal. Chim. Acta, 2009, 653, 1.

8 D. Wu, H. Xie, H. Lu, W. Li and Q. Zhang, Sensitive determination of norepinephrine, epinephrine, dopamine and 5-hydroxytryptamine by coupling HPLC with $\left[\mathrm{Ag}\left(\mathrm{HIO}_{6}\right)_{2}\right]$ 5-luminol chemiluminescence detection, Biomed. Chromatogr., 2016, 30, 1458.

9 X. Zhang, A. Rauch, H. Lee, H. Xiao, G. Rainer and N. K. Logothetis, Capillary hydrophilic interaction chromatography/mass spectrometry for simultaneous determination of multiple neurotransmitters in primate cerebral cortex, Rapid Commun. Mass Spectrom., 2007, 21, 3621.

10 A. Wojnicz, J. A. Ortiz, A. I. Casas, A. E. Freitas, M. G. Lopez and A. Ruiz-Nuno, Simultaneous determination of 8 neurotransmitters and their metabolite levels in rat brain using liquid chromatography in tandem with mass spectrometry: application to the murine Nrf2 model of depression, Clin. Chim. Acta, 2016, 453, 174.

11 G. E. De Benedetto, D. Fico, A. Pennetta, C. Malitesta, G. Nicolardi, D. D. Lofrumento and V. La Pesa, A rapid and simple method for the determination of 3,4dihydroxyphenylacetic acid, norepinephrine, dopamine, and serotonin in mouse brain homogenate by HPLC with fluorimetric detection, J. Pharm. Biomed. Anal., 2014, 98, 266.

12 A. Baranwal and P. Chandra, Clinical implications and electrochemical biosensing of monoamine neurotransmitters in body fluids, in vitro, in vivo, and ex vivo models, Biosens. Bioelectron., 2018, 121, 137.

13 E. S. Bucher and R. M. Wightman, Electrochemical analysis of neurotransmitters, Annu. Rev. Anal. Chem., 2015, 8, 239.

14 E. Katz, I. Willner and J. Wang, Electroanalytical and bioelectroanalytical systems based on metal and semiconducto nanoparticles, Electroanalysis, 2004, 16, 19.

15 C. M. Welch and R. G. Compton, The use of nanoparticles in electroanalysis: a review, Anal. Bioanal. Chem., 2004, 384, 601.

16 J. Merian, J. Gravier, F. Navarro and I. Texier, Fluorescent nanoprobes dedicated to in vivo imaging: from preclinical validations to clinical translation, Molecules, 2012, 17, 5564.

17 Y. P. Sun, B. Zhou, Y. Lin, W. Wang, K. S. Fernando, P. Pathak and P. G. Luo, Quantum-sized carbon dots for bright and colorful photoluminescence, J. Am. Chem. Soc., 2006, 128, 7756.

18 D. Pan, J. Zhang, Z. Li and M. Wu, Hydrothermal route for cutting graphene sheets into blue-luminescent graphene quantum dots, Adv. Mater., 2010, 22, 734-738.

19 H. Chen, Z. Wang, S. Zong, P. Chen, D. Zhu, L. Wu and Y. Cui, A graphene quantum dot-based FRET system for nuclear-targeted and real-time monitoring of drug delivery, Nanoscale, 2015, 7, 15477.

20 S. Y. Lim, W. Shen and Z. Q. Gao, Carbon quantum dots and their applications, Chem. Soc. Rev., 2015, 44, 362.

21 D. Zhang, L. Wen, R. Huang, H. Wang, X. Hu and D. Xing, Mitochondrial specific photodynamic therapy by rare-earth nanoparticles mediated near-infrared graphene quantum dots, Biomaterials, 2018, 153, 14.

22 B. C. M. Martindale, G. A. M. Hutton, C. A. Caputo and E. Reisner, Solar hydrogen production using carbon quantum dots and a molecular nickel catalyst, J. Am. Chem. Soc., 2015, 137, 6018.

23 P. Namdari, B. Negahdari and A. Eatemadi, Synthesis, properties and biomedical applications of carbon-based quantum dots: an updated review, Biomed. Pharmacother., 2017, 87, 209.

24 M. Li, T. Chen, J. J. Gooding and J. Liu, Review of carbon and graphene quantum dots for sensing, ACS Sens., 2019, 4, 1732.

25 I. Singh, R. Arora, H. Dhiman and R. Pahwa, Carbon Quantum Dots: Synthesis, Characterization and Biomedical Applications, Turk. J. Pharm. Sci., 2018, 15, 219.

26 X. Li, H. Wang, Y. Shimizu, A. Pyatenko, K. Kawaguchi and N. Koshizaki, Preparation of carbon quantum dots with tunable photoluminescence by rapid laser passivation in ordinary organic solvents, Chem. Commun., 2010, 47, 932.

27 C. Doñate-Buendia, R. Torres-Mendieta, A. Pyatenko, E. Falomir, M. Fernández-Alonso and G. Mínguez-Vega, Fabrication by laser irradiation in a continuous flow jet of carbon quantum dots for fluorescence imaging, ACS Omega, 2018, 3, 2735.

28 S. L. Hu, K. Y. Niu, J. Sun, J. Yang, N. Q. Zhao and X. W. Du, One-step synthesis of fluorescent carbon nanoparticles by laser irradiation, J. Mater. Chem., 2009, 19, 484.

29 H. Xu, L. Yan, V. Nguyen, Y. Yu and Y. Xu, One-step synthesis of nitrogen-doped carbon nanodots for ratiometric $\mathrm{pH}$ sensing by femtosecond laser ablation method, Appl. Surf. Sci., 2017, 414, 238.

30 (a) S. Anwar, H. Ding, M. Xu, X. Hu, Z. Li, J. Wang and M. Yan, Recent advances in synthesis, optical properties, and biomedical applications of carbon dots, ACS Appl. Bio Mater., 2019, 2, 2317; (b) K. Zhang, T. H. Lee, J. H. Cha, R. S. Varma, J.-W. Choi, H. W. Jang and M. Shokouhimehr, Two-dimensional boron nitride as a sulfur fixer for high performance rechargeable aluminum-sulfur batteries, Sci. Rep., 2019, 9, 13573; (c) K. Zhang, T. H. Lee, H. W. Jang, M. Shokouhimehr and J.-W. Choi, A hybrid energy storage mechanism of zinc hexacyanocobaltate-based metal-organic framework endowing stationary and high-performance lithium-ion storage, Electron. Mater. Lett., 2019, 15, 444; (d) K. Zhang, R. S. Varma, H. W. Jang, J. W. Choi and M. Shokouhimehr, Iron hexacyanocobaltate metal-organic framework: highly reversible and stationary electrode material with rich borders for lithium-ion batteries, $J$. Alloys Compd., 2019, 791, 911; (e) K. Zhang, T. H. Lee, B. Bubach, M. Ostadhassan, H. W. Jang, J.-W. Choi and M. Shokouhimehr, Layered metal-organic framework based on tetracyanonickelate as a cathode material for in situ Li-ion storage, $R S C A d v ., 2019,9,21363$; (f) K. Zhang, T. H. Lee, H. Noh, T. Islamoglu, O. K. Farha, H. W. Jang, J.-W. Choi and M. Shokouhimehr, Realization of lithiumion capacitors with enhanced energy density via the use 
of gadolinium hexacyanocobaltate as a cathode material, ACS Appl. Mater. Interfaces, 2019, 11, 31799; (g) K. Zhang, T. H. Lee, B. Bubach, H. W. Jang, M. Ostadhassan, J.-W. Choi and M. Shokouhimehr, Coordinating gallium hexacyanocobaltate: Prussian blue-based nanomaterial for Li-ion storage, RSC Adv., 2019, 9, 26668; (h) K. Zhang, T. H. Lee, B. Bubach, H. W. Jang, M. Ostadhassan, J.-W. Choi and M. Shokouhimehr, Graphite carbonencapsulated metal nanoparticles derived from Prussian blue analogs growing on natural loofa as cathode materials for rechargeable aluminum-ion batteries, Sci. Rep., 2019, 9, 13665.

31 M. Liu, Y. Xu, F. Niu, J. J. Gooding and J. Liu, Carbon quantum dots directly generated from electrochemical oxidation of graphite electrodes in alkaline alcohols and the applications for specific ferric ion detection and cell imaging, Analyst, 2016, 141, 2657.

32 J. Deng, Q. Lu, N. Mi, H. Li, M. Liu, M. Xu and S. Yao, Electrochemical synthesis of carbon nanodots directly from alcohols, Chem.-Eur. J., 2014, 20, 4993.

33 X. Xu, R. Ray, Y. Gu, H. J. Ploehn, L. Gearheart, K. Raker and W. A. Scrivens, Electrophoretic analysis and purification of fluorescent single-walled carbon nanotube fragments, $J$. Am. Chem. Soc., 2004, 126, 12736.

34 N. Arora and N. N. Sharma, Arc discharge synthesis of carbon nanotubes: Comprehensive review, Diam. Relat. Mater., 2014, 50, 135.

35 S. Y. Park, H. U. Lee, E. S. Park, S. C. Lee, J. W. Lee, S. W. Jeong and J. Lee, Photoluminescent green carbon nanodots from food-waste-derived sources: large-scale synthesis, properties, and biomedical applications, ACS Appl. Mater. Interfaces, 2014, 6, 3365.

36 Y. Zhang, M. Park, H. Y. Kim, B. Ding and S. J. Park, A facile ultrasonic-assisted fabrication of nitrogen-doped carbon dots/BiOBr up-conversion nanocomposites for visible light photocatalytic enhancements, Sci. Rep., 2017, 7, 45086.

37 Z. Ma, H. Ming, H. Huang, Y. Liu and Z. Kang, One-step ultrasonic synthesis of fluorescent $\mathrm{N}$-doped carbon dots from glucose and their visible-light sensitive photocatalytic ability, New J. Chem., 2012, 36, 861.

38 H. Dang, L. K. Huang, Y. Zhang, C. F. Wang and S. Chen, Large-scale ultrasonic fabrication of white fluorescent carbon dots, Ind. Eng. Chem. Res., 2016, 55, 5335.

39 S. C. Ray, A. Saha, N. R. Jana and R. Sarkar, Fluorescent carbon nanoparticles: synthesis, characterization, and bioimaging application, J. Phys. Chem. C, 2009, 113, 18546.

40 Z. A. Qiao, Y. Wang, Y. Gao, H. Li, T. Dai, Y. Liu and Q. Huo, Commercially activated carbon as the source for producing multicolor photoluminescent carbon dots by chemical oxidation, Chem. Commun., 2010, 46, 8812.

$41 \mathrm{H}$. Peng and J. Travas-Sejdic, Simple aqueous solution route to luminescent carbogenic dots from carbohydrates, Chem. Mater., 2009, 21, 5563.

42 Q. Zhang, X. Sun, H. Ruan, K. Yin and H. Li, Production of yellow-emitting carbon quantum dots from fullerene carbon soot, Sci. China Mater., 2017, 60, 141.
43 B. Chen, F. Li, S. Li, W. Weng, H. Guo, T. Guo and S. You, Large scale synthesis of photoluminescent carbon nanodots and their application for bioimaging, Nanoscale, 2013, 5, 1967.

44 H. Wang, G. Ning, X. He, X. Ma, F. Yang, Z. Xu and Y. Li, Carbon quantum dots derived by direct carbonization of carbonaceous microcrystals in mesophase pitch, Nanoscale, 2018, 10, 21492.

45 C. B. Ma, Z. T. Zhu, H. X. Wang, X. Huang, X. Zhang, X. Qi and Y. A. Han, general solid-state synthesis of chemicallydoped fluorescent graphene quantum dots for bioimaging and optoelectronic applications, Nanoscale, 2015, 7, 10162.

46 H. Zhu, X. Wang, Y. Li, Z. Wang, F. Yang and X. Yang, Microwave synthesis of fluorescent carbon nanoparticles with electrochemiluminescence properties, Chem. Commun., 2009, 34, 5118.

47 D. Rodríguez-Padrón, M. Algarra, L. A. Tarelho, J. Frade, A. Franco, G. de Miguel and R. Luque, Catalyzed microwave-assisted preparation of carbon quantum dots from lignocellulosic residues, ACS Sustain. Chem. Eng., 2018, 6, 7200.

48 P. Yang, Z. Zhu, M. Chen, W. Chen and X. Zhou, Microwaveassisted synthesis of xylan-derived carbon quantum dots for tetracycline sensing, Opt. Mater., 2018, 85, 329.

49 N. R. Ko, M. Nafiujjaman, K. Cherukula, S. J. Lee, S. J. Hong, H. N. Lim and I. K. Kwon, Microwave-Assisted Synthesis of Biocompatible Silk Fibroin-Based Carbon Quantum Dots, Part. Part. Syst. Char., 2018, 35, 1700300.

50 C. W. Lai, Y. H. Hsiao, Y. K. Peng and P. T. Chou, Facile synthesis of highly emissive carbon dots from pyrolysis of glycerol; gram scale production of carbon dots $/ \mathrm{mSiO}_{2}$ for cell imaging and drug release, J. Mater. Chem., 2012, 22, 14403.

51 Y. Yang, D. Wu, S. Han, P. Hu and R. Liu, Bottom-up fabrication of photoluminescent carbon dots with uniform morphology via a soft-hard template approach, Chem. Commun., 2013, 49, 4920.

52 M. M. Titirici and M. Antonietti, Chemistry and materials options of sustainable carbon materials made by hydrothermal carbonization, Chem. Soc. Rev., 2010, 39, 103.

53 T. Shen, Q. Wang, Z. Guo, J. Kuang and W. Cao, Hydrothermal synthesis of carbon quantum dots using different precursors and their combination with $\mathrm{TiO}_{2}$ for enhanced photocatalytic activity, Ceram. Int., 2018, 44, 11828.

54 C. Zhao, Y. Jiao, J. Hua, J. Yang and Y. Yang, Hydrothermal synthesis of nitrogen-doped carbon quantum dots as fluorescent probes for the detection of dopamine, $J$. Fluoresc., 2018, 28, 269.

55 S. K. Bhunia, A. Saha, A. R. Maity, S. C. Ray and N. R. Jana, Carbon nanoparticle-based fluorescent bioimaging probes, Sci. Rep., 2013, 3, 1473.

56 Q. Wang, X. Huang, Y. Long, X. Wang, H. Zhang, R. Zhu and H. Zheng, Hollow luminescent carbon dots for drug delivery, Carbon, 2013, 59, 192.

57 X. Zhang, C. Wei, Y. Li and D. Yu, Shining luminescent graphene quantum dots: synthesis, physicochemical 
properties, and biomedical applications, TrAC, Trends Anal. Chem., 2019, 116, 109.

58 M. He, X. Guo, J. Huang, H. Shen, Q. Zeng and L. Wang, Mass production of tunable multicolor graphene quantum dots from an energy resource of coke by a onestep electrochemical exfoliation, Carbon, 2018, 140, 508.

59 Y. Fu, G. Gao and J. Zhi, Electrochemical synthesis of multicolor fluorescent $\mathrm{N}$-doped graphene quantum dots as a ferric ion sensor and their application in bioimaging, J. Mater. Chem. B, 2019, 7, 1494.

60 S. Zhuo, M. Shao and S. T. Lee, Upconversion and downconversion fluorescent graphene quantum dots: ultrasonic preparation and photocatalysis, ACS Nano, 2012, 6, 1059.

61 J. Peng, W. Gao, B. K. Gupta, Z. Liu, R. Romero-Aburto, L. Ge and S. A. Vithayathil, Graphene quantum dots derived from carbon fibers, Nano Lett., 2012, 12, 844.

62 D. Iannazzo, A. Pistone, S. Ferro, L. De Luca, A. M. Monforte, R. Romeo and C. Panecouque, Graphene Quantum Dots Based Systems As HIV Inhibitors, Bioconjugate Chem., 2018, 29, 3084.

63 L. A. Ponomarenko, F. Schedin, M. I. Katsnelson, R. Yang, E. W. Hill, K. S. Novoselov and A. K. Geim, Chaotic Dirac billiard in graphene quantum dots, Science, 2008, 320, 356.

64 J. Lee, K. Kim, W. I. Park, B. H. Kim, J. H. Park, T. H. Kim and Y. Hwang, Uniform graphene quantum dots patterned from self-assembled silica nanodots, Nano Lett., 2012, 12, 6078.

65 B. Sapkota, A. Benabbas, H. Y. G. Lin, W. Liang, P. Champion and M. Wanunu, Peptide-decorated tunablefluorescence graphene quantum dots, ACS Appl. Mater. Interfaces, 2017, 9, 9378.

66 A. Biswas, P. Khandelwal, R. Das, G. Salunke, A. Alam, S. Ghorai and P. Poddar, Oxidant mediated one-step complete conversion of multi-walled carbon nanotubes to graphene quantum dots and their bioactivity against mammalian and bacterial cells, J. Mater. Chem. B, 2017, 5, 785.

67 G. L. Hong, H. L. Zhao, H. H. Deng, H. J. Yang, H. P. Peng, Y. H. Liu and W. Chen, Fabrication of ultra-small monolayer graphene quantum dots by pyrolysis of trisodium citrate for fluorescent cell imaging, Int. J. Nanomed., 2018, 13, 4807.

68 T. Gao, X. Wang, L. Y. Yang, H. He, X. X. Ba, J. Zhao and Y. Liu, Red, yellow, and blue luminescence by graphene quantum dots: syntheses, mechanism, and cellular imaging, ACS Appl. Mater. Interfaces, 2017, 9, 24846.

69 S. Kaciulis, A. Mezzi, P. Soltani, R. Pizzoferrato, E. Ciotta and P. Prosposito, Graphene quantum dots obtained by unfolding fullerene, Thin Solid Films, 2019, 673, 19.

70 J. Lu, P. S. E. Yeo, C. K. Gan, P. Wu and K. P. Loh, Transforming $\mathrm{C}_{60}$ molecules into graphene quantum dots, Nat. Nanotechnol., 2011, 6, 247.

71 W. Li, M. Li, Y. Liu, D. Pan, Z. Li, L. Wang and M. Wu, Three Minute Ultrarapid Microwave-Assisted Synthesis of Bright Fluorescent Graphene Quantum Dots for Live Cell
Staining and White LEDs, ACS Appl. Nano Mater., 2018, 1, 1623.

72 M. K. Kumawat, M. Thakur, R. B. Gurung and R. Srivastava, Graphene quantum dots from mangifera indica: application in near-infrared bioimaging and intracellular nanothermometry, ACS Sustain. Chem. Eng., 2017, 5, 1382.

73 M. Yousaf, H. Huang, P. Li, C. Wang and Y. Yang, Fluorine functionalized graphene quantum dots as inhibitor against hIAPP amyloid aggregation, ACS Chem. Neurosci., 2017, 8, 1368.

74 S. Ge, J. He, C. Ma, J. Liu, F. Xi and X. Dong, One-step synthesis of boron-doped graphene quantum dots for fluorescent sensors and biosensor, Talanta, 2019, 199, 581.

75 K. B. Ko, Z. Khurelbaatar, C. J. Choi, C. H. Hong and T. V. Cuong, Transparent and flexible ultraviolet photoconductors based on solution-processed graphene quantum dots on reduced graphene oxide films, Mater. Res. Bull., 2017, 91, 49.

76 R. Li, Y. Liu, Z. Li, J. Shen, Y. Yang, X. Cui and G. Yang, Bottom-up fabrication of single-layered nitrogen-doped graphene quantum dots through Intermolecular carbonization arrayed in a 2D plane, Chem.-Eur. J., 2016, 22, 272.

77 S. Gao, L. Tang, J. Xiang, R. Ji, S. K. Lai, S. Yuan and S. P. Lau, Facile preparation of sulphur-doped graphene quantum dots for ultra-high performance ultraviolet photodetectors, New J. Chem., 2017, 41, 10447.

78 X. Ke, C. Bittencourt and G. Van Tendeloo, Possibilities and limitations of advanced transmission electron microscopy for carbon-based nanomaterials, Beilstein J. Nanotechnol., 2015, 6, 1541.

79 X. Jia, J. Campos-Delgado, M. Terrones, V. Meunier and M. S. Dresselhaus, Graphene edges: a review of their fabrication and characterization, Nanoscale, 2011, 3, 86.

80 B. Guo, Y. Zuo, Y. Shi, T. Han and M. Lanza, Transmission electron microscopy-based statistical analysis of commercially available graphene oxide quantum dots, Cryst. Res. Technol., 2020, 1900231.

81 Y. Fang, S. Guo, D. Li, C. Zhu, W. Ren, S. Dong and E. Wang, Easy synthesis and imaging applications of cross-linked green fluorescent hollow carbon nanoparticles, Acs Nano, 2011, 6, 400.

82 R. Liu, D. Wu, X. Feng and K. Müllen, Bottom-up fabrication of photoluminescent graphene quantum dots with uniform morphology, J. Am. Chem. Soc., 2011, 133, 15221.

83 J. Shen, Y. Zhu, X. Yang and C. Li, Graphene quantum dots: emergent nanolights for bioimaging, sensors, catalysis and photovoltaic devices, Chem. Commun., 2012, 48, 3686.

84 S. Jing, Y. Zhao, R. C. Sun, L. Zhong and X. Peng, Facile and High-Yield Synthesis of Carbon Quantum Dots from Biomass-Derived Carbons at Mild Condition, ACS Sustain. Chem. Eng., 2019, 7, 7833.

85 S. H. Lee, D. Y. Kim, J. Lee, S. B. Lee, H. Han, Y. Y. Kim and O. O. Park, Synthesis of single-crystalline hexagonal graphene quantum dots from solution chemistry, Nano Lett., 2019, 19, 5437. 
86 P. Zuo, X. Lu, Z. Sun, Y. Guo and H. A. He, review on syntheses, properties, characterization and bioanalytical applications of fluorescent carbon dots, Microchim. Acta, 2016, 183, 519.

87 D. Barmpakos, A. Segkos, C. Tsamis and G. Kaltsas, Enhancement Of PEDOT: PSS Seebeck coefficient using Carbon-quantum-dot-based nanocomposite materials: application to inkjet printing on flexible substrate, in 20th International Conference on Solid-State Sensors, Actuators and Microsystems \& Eurosensors, 2515, 2019.

88 D. Iannazzo, A. Pistone, M. Salamò, S. Galvagno, R. Romeo, S. V. Giofré and A. Di Pietro, Graphene quantum dots for cancer targeted drug delivery, Int. J. Pharm., 2017, 518, 185.

89 X. T. Zheng, A. Ananthanarayanan, K. Q. Luo and P. Chen, Glowing graphene quantum dots and carbon dots: properties, syntheses, and biological applications, Small, 2015, 11, 1620.

90 S. Zhu, Y. Song, X. Zhao, J. Shao, J. Zhang and B. Yang, The photoluminescence mechanism in carbon dots (graphene quantum dots, carbon nanodots, and polymer dots): current state and future perspective, Nano Res., 2015, 8, 355.

91 A. B. Bourlinos, A. Stassinopoulos, D. Anglos, R. Zboril, V. Georgakilas and E. P. Giannelis, Photoluminescent carbogenic dots, Chem. Mater., 2008, 20, 4539.

92 G. Rajender, U. Goswami and P. K. Giri, Solvent dependent synthesis of edge-controlled graphene quantum dots with high photoluminescence quantum yield and their application in confocal imaging of cancer cells, J. Colloid Interface Sci., 2019, 541, 387.

93 W. Chen, D. Li, L. Tian, W. Xiang, T. Wang, W. Hu and Z. Dai, Synthesis of graphene quantum dots from natural polymer starch for cell imaging, Green Chem., 2018, 20, 4438.

94 S. Thambiraj and R. Shankaran, Green synthesis of highly fluorescent carbon quantum dots from sugarcane bagasse pulp, Appl. Surf. Sci., 2016, 390, 435.

95 E. Rezaii and M. Mahkam, Synthesis of layered lipophilic graphene quantum dot over $\mathrm{Fe@} \mathrm{MgO} \mathrm{catalyst,} \mathrm{Mater.}$ Chem. Phys., 2019, 232, 65.

96 M. Algarra, A. González-Calabuig, K. Radotić, D. Mutavdzic, C. O. Ania, J. M. Lázaro-Martínez and M. Del Valle, Enhanced electrochemical response of carbon quantum dot modified electrodes, Talanta, 2018, 178, 679.

97 J. B. Wu, M. L. Lin, X. Cong, H. N. Liu and P. H. Tan, Raman spectroscopy of graphene-based materials and its applications in related devices, Chem. Soc. Rev., 2018, 47, 1822.

98 E. Dervishi, Z. Ji, H. Htoon, M. Sykora and S. K. Doorn, Raman spectroscopy of bottom-up synthesized graphene quantum dots: size and structure dependence, Nanoscale, 2019, 11, 16571.

99 D. Kumar, K. Singh, V. Verma and H. S. Bhatti, Synthesis and characterization of carbon quantum dots from orange juice, J. Bionanoscience, 2014, 8, 274.

100 M. J. Molaei, A review on nanostructured carbon quantum dots and their applications in biotechnology, sensors, and chemiluminescence, Talanta, 2019, 196, 456.
101 L. Lin, M. Rong, F. Luo, D. Chen, Y. Wang and X. Chen, Luminescent graphene quantum dots as new fluorescent materials for environmental and biological applications, TrAC, Trends Anal. Chem., 2014, 54, 83.

102 J. Fang, Y. Liu, Y. Chen, D. Ouyang, G. Yang and T. Yu, Graphene quantum dots-gated hollow mesoporous carbon nanoplatform for targeting drug delivery and synergistic chemo-photothermal therapy, Int. J. Nanomed., 2018, 13, 5991.

103 S. Ghosh, K. Ghosal, S. A. Mohammad and K. Sarkar, Dendrimer functionalized carbon quantum dot for selective detection of breast cancer and gene therapy, Chem. Eng. J., 2019, 373, 468.

104 Q. Duan, Y. Ma, M. Che, B. Zhang, Y. Zhang, Y. Li and S. Sang, Fluorescent carbon dots as carriers for intracellular doxorubicin delivery and track, J. Drug Deliv. Sci. Technol., 2019, 49, 527.

105 W. Q. Li, Z. Wang, S. Hao, L. Sun, M. Nisic, G. Cheng and S. Y. Zheng, Mitochondria-based aircraft carrier enhances in vivo imaging of carbon quantum dots and delivery of anticancer drug, Nanoscale, 2018, 10, 3744.

106 G. Wang, A. Xu, P. He, Q. Guo, Z. Liu, Z. Wang and Y. Wang, Green preparation of lattice phosphorus doped graphene quantum dots with tunable emission wavelength for bioimaging, Mater. Lett., 2019, 242, 156.

107 H. Singh, S. Sreedharan, K. Tiwari, N. H. Green, C. Smythe, S. K. Pramanik and A. Das, Two photon excitable graphene quantum dots for structured illumination microscopy and imaging applications: lysosome specificity and tissuedependent imaging, Chem. Commun., 2019, 55, 521.

108 M. Kovacova, Z. M. Marković, P. Humpolíček, M. Mičušík, H. Švajdlenková, A. Kleinová and M. Lehocky, Carbon quantum dots modified polyurethane nanocomposite as effective photocatalytic and antibacterial agents, ACS Biomater. Sci. Eng., 2018, 4, 3983.

109 S. Chen, Y. Quan, Y. L. Yu and J. H. Wang, Graphene quantum dot/silver nanoparticle hybrids with oxidase activities for antibacterial application, ACS Biomater. Sci. Eng., 2017, 3, 313.

110 Y. Wang, W. Kong, L. Wang, J. Z. Zhang, Y. Li, X. Liu and Y. Li, Optimizing oxygen functional groups in graphene quantum dots for improved antioxidant mechanism, Phys. Chem. Chem. Phys., 2019, 21, 1336.

111 L. A. Chunduri, A. Kurdekar, S. Patnaik, B. V. Dev, T. M. Rattan and V. Kamisetti, Carbon quantum dots from coconut husk: evaluation for antioxidant and cytotoxic activity, Mater. Focus, 2016, 5, 55.

112 S. Ahmadian-Fard-Fini, D. Ghanbari, O. Amiri and M. Salavati-Niasari, Electro-spinning of cellulose acetate nanofibers/Fe/carbon dot as photoluminescence sensor for mercury (II) and lead (II) ions, Carbohydr. Polym., 2020, 229, 115428.

113 C. Ruiz-Palomero, M. L. Soriano, S. Benítez-Martínez and M. Valcarcel, Photoluminescent sensing hydrogel platform based on the combination of nanocellulose and S, N-codoped graphene quantum dots, Sens. Actuators, B, 2017, 245, 946. 
114 Q. Zhang, C. Song, T. Zhao, H. W. Fu, H. Z. Wang, Y. J. Wang and D. M. Kong, Photoluminescent sensing for acidic amino acids based on the disruption of graphene quantum dots/europium ions aggregates, Biosens. Bioelectron., 2015, 65, 204.

115 H. Sun, L. Wu, W. Wei and X. Qu, Recent advances in graphene quantum dots for sensing, Mater. Today, 2013, 16, 433.

116 X. You, W. Lin, H. Wu, Y. Dong and Y. Chi, Carbon dot capped gold nanoflowers for electrochemiluminescent aptasensor of thrombin, Carbon, 2018, 127, 653.

117 G. Jie, Q. Zhou and G. Jie, Graphene quantum dots-based electrochemiluminescence detection of DNA using multiple cycling amplification strategy, Talanta, 2019, 194, 658.

118 K. Tian, D. Li, T. Tang, F. Nie, Y. Zhou, J. Du and J. A. Zheng, Novel electrochemiluminescence resonance energy transfer system of luminol-graphene quantum dot composite and its application in $\mathrm{H}_{2} \mathrm{O}_{2}$ detection, Talanta, 2018, 185, 446.

119 C. S. Lim, K. Hola, A. Ambrosi, R. Zboril and M. Pumera, Graphene and carbon quantum dots electrochemistry, Electrochem. Commun., 2015, 52, 75.

120 Q. Xiang, J. Huang, H. Huang, W. Mao and Z. A. Ye, labelfree electrochemical platform for the highly sensitive detection of hepatitis B virus DNA using graphene quantum dots, $R S C A d v ., 2018,8,1820$.

121 S. K. Tuteja, R. Chen, M. Kukkar, C. K. Song, R. Mutreja, S. Singh and C. R. Suri, A label-free electrochemical immunosensor for the detection of cardiac marker using graphene quantum dots (GQDs), Biosens. Bioelectron., 2016, 86, 548.

122 S. Zhao, C. Li, J. Liu, N. Liu, S. Qiao, Y. Han and Z. Kang, Carbon quantum dots $/ \mathrm{SnO}_{2}-\mathrm{Co}_{3} \mathrm{O}_{4}$ composite for highly efficient electrochemical water oxidation, Carbon, 2015, 92, 64.

123 Q. Huang, S. Hu, H. Zhang, J. Chen, Y. He, F. Li and Y. Lin, Carbon dots and chitosan composite film based biosensor for the sensitive and selective determination of dopamine, Analyst, 2013, 138, 5417.

124 Q. Huang, H. Zhang, S. Hu, F. Li, W. Weng, J. Chen and $\mathrm{X}$. Bao, A sensitive and reliable dopamine biosensor was developed based on the Au@ carbon dots-chitosan composite film, Biosens. Bioelectron., 2014, 52, 277.

125 S. Hu, Q. Huang, Y. Lin, C. Wei, H. Zhang, W. Zhang and A. Hao, Reduced graphene oxide-carbon dots composite as an enhanced material for electrochemical determination of dopamine, Electrochim. Acta, 2014, 130, 805.

126 G. Jiang, T. Jiang, H. Zhou, J. Yao and X. Kong, Preparation of $\mathrm{N}$-doped carbon quantum dots for highly sensitive detection of dopamine by an electrochemical method, RSC Adv., 2015, 5, 9064.

127 Y. Jiang, B. Wang, F. Meng, Y. Cheng and C. Zhu, Microwave-assisted preparation of $\mathrm{N}$-doped carbon dots as a biosensor for electrochemical dopamine detection, $J$. Colloid Interface Sci., 2015, 452, 199.
128 Q. Li, Z. Xu, W. Tang and Y. Wu, Determination of dopamine with a modified carbon dot electrode, Anal. Lett., 2015, 48, 2040.

129 Q. Huang, X. Lin, C. Lin, Y. Zhang, S. Hu and C. Wei, A high performance electrochemical biosensor based on $\mathrm{Cu} 2 \mathrm{O}-$ carbon dots for selective and sensitive determination of dopamine in human serum, RSC Adv., 2015, 5, 54102.

130 L. Wang, X. Chen, C. Liu and W. Yang, Non-enzymatic acetylcholine electrochemical biosensor based on flowerlike NiAl layered double hydroxides decorated with carbon dots, Sens. Actuators, B, 2016, 233, 199.

131 T. C. Canevari, M. Nakamura, F. H. Cincotto, F. M. de Melo and H. E. Toma, High performance electrochemical sensors for dopamine and epinephrine using nanocrystalline carbon quantum dots obtained under controlled chronoamperometric conditions, Electrochim. Acta, 2016, 209, 464.

132 X. Zhuang, H. Wang, T. He and L. Chen, Enhanced voltammetric determination of dopamine using a glassy carbon electrode modified with ionic liquidfunctionalized graphene and carbon dots, Microchim. Acta, 2016, 183, 3177.

133 J. Chen, P. He, H. Bai, S. He, T. Zhang, X. Zhang and F. Dong, Poly ( $\beta$-cyclodextrin)/carbon quantum dots modified glassy carbon electrode: preparation, characterization and simultaneous electrochemical determination of dopamine, uric acid and tryptophan, Sens. Actuators, B, 2017, 252, 9.

134 J. Fang, Z. Xie, G. Wallace and X. Wang, Co-deposition of carbon dots and reduced graphene oxide nanosheets on carbon-fiber microelectrode surface for selective detection of dopamine, Appl. Surf. Sci., 2017, 412, 131.

135 N. R. Devi, T. V. Kumar and A. K. Sundramoorthy, Electrochemically exfoliated carbon quantum dots modified electrodes for detection of dopamine neurotransmitter, J. Electrochem. Soc., 2018, 165, G3112.

136 M. Algarra, A. González-Calabuig, K. Radotić, D. Mutavdzic, C. O. Ania, J. M. Lázaro-Martínez and M. Del Valle, Enhanced electrochemical response of carbon quantum dot modified electrodes, Talanta, 2018, 178, 679.

137 M. L. Yola and N. Atar, Development of molecular imprinted sensor including graphitic carbon nitride/Ndoped carbon dots composite for novel recognition of epinephrine, Compos. B Eng., 2019, 175, 107113.

138 P. Pang, F. Yan, H. Li, H. Li, Y. Zhang, H. Wang and W. Yang, Graphene quantum dots and Nafion composite as an ultrasensitive electrochemical sensor for the detection of dopamine, Anal. Methods, 2016, 8, 4912.

139 A. Chen, C. Zhao, Y. Yu and J. Yang, Graphene quantum dots derived from carbon fibers for oxidation of dopamine, J. Wuhan Univ. Technol., Mater. Sci. Ed., 2016, 31, 1294.

140 E. Habibi and H. Heidari, Renewable surface carbon-composite electrode bulk modified with $\mathrm{GQD} \mathrm{RuCl}_{3}$ nano-composite for high sensitive detection of l-tyrosine, Electroanalysis, 2016, 28, 2559. 
141 M. Hasanzadeh, A. Karimzadeh, N. Shadjou, A. Mokhtarzadeh, L. Bageri, S. Sadeghi and S. Mahboob, Graphene quantum dots decorated with magnetic nanoparticles: Synthesis, electrodeposition, characterization and application as an electrochemical sensor towards determination of some amino acids at physiological pH, Mater. Sci. Eng., C, 2016, 68, 814.

142 M. L. Yola and N. Atar, Functionalized graphene quantum dots with bi-metallic nanoparticles composite: sensor application for simultaneous determination of ascorbic acid, dopamine, uric acid and tryptophan, J. Electrochem. Soc., 2016, 163, B718.

143 Y. Li, Y. Jiang, T. Mo, H. Zhou, Y. Li and S. Li, Highly selective dopamine sensor based on graphene quantum dots self-assembled monolayers modified electrode, $J$. Electroanal. Chem., 2016, 767, 84.

144 L. Ruiyi, Q. Sili, L. Zhangyi, L. Ling and L. Zaijun, Histidinefunctionalized graphene quantum dot-graphene microaerogel based voltammetric sensing of dopamine, Sens. Actuators, B, 2017, 250, 372.

145 S. Ben Aoun, Nanostructured carbon electrode modified with N-doped graphene quantum dots-chitosan nanocomposite: a sensitive electrochemical dopamine sensor, R. Soc. Open Sci., 2017, 4, 171199.

$146 \mathrm{~S}$. Baluta, A. Lesiak and J. Cabaj, Graphene quantum dots-based electrochemical biosensor for catecholamine neurotransmitters detection, Electroanalysis, 2018, 30, 1781.

147 N. Shadjou, M. Hasanzadeh and F. Talebi, Graphene quantum dots incorporated into $\beta$-cyclodextrin: a novel polymeric nanocomposite for non-enzymatic sensing of Ltyrosine at physiological pH, J. Anal. Chem., 2018, 73, 602.
148 M. L. Yola and N. Atar, A novel detection approach for serotonin by graphene quantum dots/two-dimensional (2D) hexagonal boron nitride nanosheets with molecularly imprinted polymer, Appl. Surf. Sci., 2018, 458, 648.

149 S. Zheng, R. Huang, X. Ma, J. Tang, Z. Li, X. Wang and J. Wang, A highly sensitive dopamine sensor based on graphene quantum dots modified glassy carbon Eelectrode, Int. J. Electrochem. Sci., 2018, 13, 5723.

150 H. Beitollahi, Z. Dourandish, M. R. Ganjali and S. Shakeri, Voltammetric determination of dopamine in the presence of tyrosine using graphite screen-printed electrode modified with graphene quantum dots, Ionics, 2018, 24, 4023.

151 J. Tashkhourian, S. F. Nami-Ana and M. Shamsipur, Designing a modified electrode based on graphene quantum dot-chitosan application to electrochemical detection of epinephrine, J. Mol. Liq., 2018, 266, 548.

152 A. Fajardo, D. Tapia, J. Pizarro, R. Segura and P. Jara, Determination of norepinephrine using a glassy carbon electrode modified with graphene quantum dots and gold nanoparticles by square wave stripping voltammetry, $J$. Appl. Electrochem., 2019, 49, 423.

153 V. Vinoth, L. N. Natarajan, R. V. Mangalaraja, H. Valdés and S. Anandan, Simultaneous electrochemical determination of dopamine and epinephrine using gold nanocrystals capped with graphene quantum dots in a silica network, Microchim. Acta, 2019, 186, 681.

154 P. Mohammadzadeh Jahani, M. Jafari, V. K. Gupta and S. Agarwal, Graphene quantum dots/ionic liquid-modified carbon paste electrode-based sensor for simultaneous voltammetric determination of norepinephrine and acetylcholine, Int. J. Electrochem. Sci., 2020, 15, 947. 